УДК 512.662 .5

N. A. Kachanovsky (Inst. Math. Nat. Acad. Sci. Ukraine, Kyiv)

\title{
ELEMENTS OF A NON-GAUSSIAN ANALYSIS ON SPACES OF FUNCTIONS OF INFINITELY MANY VARIABLES
}

We present a review of some results of a non-Gaussian analysis under the biorthogonal approach and consider elements of an analysis associated with the generalized Meixner measure. The main objects of our interest are stochastic integrals, operators of stochastic differentiation, elements of a Wick calculus, and related topics.

Наведено огляд деяких результатів негауссівського аналізу при біортогональному підході та розглянуто елементи аналізу, пов'язаного з узагальненою мірою Майкснера. Основними об'єктами, що розглядаються, є стохастичні інтеграли, оператори стохастичного диференціювання, елементи віківського числення та споріднені питання.

Introduction. The development of modern mathematical branches of science, in particular, of mathematical physics and stochastic analysis, requires to construct an extensive theory of generalized functions of infinitely many variables. One direction of this theory that consists in the study of test and generalized function spaces as infinite tensor products of one-dimensional spaces was triggered by Yu. M. Berezansky, Yu. G. Kondratiev, and Yu. S. Samoilenko (see [21, 85, 86]). Afterwards, independently of one another, Yu. G. Kondratiev [81-83] (see also [87]) and T. Hida (see [54, 55]) constructed a detailed theory of generalized functions of infinitely many variables with special spaces of test and generalized functions such that the pairing between elements of these spaces is generated by integration with respect to the Gaussian measure.

On the other hand, approximately at the same time, Yu. M. Berezansky and disciples were constructing a theory of generalized functions (see [14, 21, 92]) related to product measures. This theory is less detailed than the "Gaussian" one, but is more general because deals with a dual pairing generated by integration with respect to a non-Gaussian measure.

With regard for these researches, it is natural to try to construct a theory of generalized functions of infinitely many variables, which deals with a general, as far as possible, dual pairing, but is similar by detalization of results to the "Gaussian" theory (for this theory we use the term a non-Gaussian infinite-dimensional analysis). The first works in this direction are the papers of Y. Ito and I. Kubo [59, 60], in which some results of the Gaussian analysis are transfered to the case where the Gaussian measure is replaced by the Poissonian measure. The posterior development of the above-mentioned theory ocured in different directions. For example, in 1991, Yu. M. Berezansky [15] offered to construct orthogonal decompositions of "base" spaces (these spaces generate dual pairings) using families of commuting self-adjoint operators. Typical examples of works in this direction are $[18,95]$. Another direction is based on the idea of Yu. G. Kondratiev to use biorthogonal systems [30] (that consist of generalized Appell polynomials and (generalized) functions dual to these polynomials) as orthogonal bases in spaces of test and generalized functions. This idea was realized by Yu. G. Kondratiev and his colleagues first for the case of so-called smooth twice analytic measures [7, 8, 90], later for more general analytic nondegenerate measures [88, 91]. After that different researches and generalizations were executed by many specialists; in particular, by G. F. Us [116], by Yu. M. Berezansky and Yu. G. Kondratiev [16], by Yu. M. Berezansky [12], by the author, see, e.g., [67-69, 74, 75], by V. A. Tesko [112], by Yu. M. Berezansky and V. A. Tesko 
[22-24], by E. Yablonsky [118] etc. Moreover, it was ascertained that insignificant modifications of a biorthogonal approach to the construction of a non-Gaussian analysis give a possibility to extend an area of possible applications. For example, it is possible to use results of this approach in order to construct elements of a so-called coloured noise analysis, see, e.g., $[65,78,117]$.

Let us say several words about nonclassical examples of application of the "biorthogonal analysis". In the end of 1990-es, in the papers [84, 89], the infinite-dimensional analog of the so-called gamma-measure was constructed and investigated. One can show (see, e.g., [76]) that this measure satisfies all requirements of the "biorthogonal analysis". Moreover, the results of the "gamma analysis" that are closely related to a structure and properties of the gamma-measure and cannot be obtained from the "biorthogonal theory" (for example, the construction of the extended stochastic integral on the space of squareintegrable functions), are naturally coordinated with general results of the "biorthogonal analysis". This example is not unique. In 2002, Yu. M. Berezansky [13] constructed and studied an infinite-dimensional analog of the Pascal measure; in 2003, E. W. Lytvynov $[96,97]$ considered infinite-dimensional analogs of so-called Meixner-type measures (to this type belong the Gaussian, Poissonian, Pascal, Meixner measures and gammameasures, see [98]) and constructed elements of the corresponding analysis basingn on the so-called Jacobi fields theory, see, e.g., [19]; in 2005, I. V. Rodionova [108] considered a wide class of infinite-dimensional Meixner-type measures (these measures are realized as a so-called generalized Meixner measure, which subject to parameters can be Gaussian, Poissonian, gamma-measure etc.); her research consists in the generalization of results presented in [97]. It is worth noting that the white noise in [108] is not a Lévy white noise, generally speaking (not time-homogeneous). In the investigations of [96, 97, 108], an important role belongs to the so-called extended Fock space [84, 20], which naturally arises in the "Meixner analysis" and, in fact, is the interacting Fock space $[3,58]$. All mentioned measures satisfy all requirements of the "biorthogonal analysis"; and specific results related to properties of these measures are naturally coordinated with the results of the "biorthogonal analysis". Moreover, in order to obtain these specific results it is convenient to use a "tooling" that is developed in the "biorthogonal analysis".

During recent years, an analysis associated with the Gamma, Pascal and Meixner measures and the corresponding white noises (including stochastic integration theory) became the object of investigation of many authors. In particular, in [1], Lévy processes on the Lie algebra $\operatorname{sl}(2, \mathbb{R})$ were investigated, components of these processes are classical Lévy processes on $\mathbb{R}$ corresponding to Meixner classes; in [4], the stochastic integral was introduced and studied for a wide class of stochastic processes and it was proved in [2] that the results of [4] can be applied in the "Meixner analysis"; in [52, 53], the stochastic integration theory with applications was constructed for Meixner processes and its generalizations; in [113 - 115], some properties of gamma-processes were studied; in [5], all Meixner classes within a quantum white noise context were considered from a general point of view.

On the other hand, many specialists study a (non-Gaussian, generally speaking) analysis on the so-called Hida (e.g., [32, 34, 56]), Kondratiev (e.g., [7, 8, 12, 24, 67, 68, $88,90,91,112]$ ) and another similar spaces of test and generalized functions (and on the corresponding weighted Fock spaces). Such an analysis includes a stochastic integration theory, a Wick calculus and different applications (including a theory of normally ordered 
white noise equations or, in another terminology, of stochastic equations with Wick-type nonlinearities). Thereupon we refer, in particular, to the papers $[8,27-29,32,34$, $61,62,90,93,102-106]$. One of tasks in these investigations consists in the study of properties of different operators (including stochastic integrals and stochastic derivatives) and operations (e.g., of a Wick multiplication) subject to the particular spaces under consideration. For example, in [32-34], stochastic integrals with respect to a wide class of Lévy processes on Hida spaces are studied and the corresponding Wick calculus is developed; the constructions in these works are based on the so-called power jump processes [101].

So, the problem of development and improvement of the biorthogonal approach to the construction of a non-Gaussian analysis (in particular, the construction and study of stochastic integrals and operators of stochastic differentiation, of a Wick calculus; improvement of the "tooling" etc.), just as the problem of construction and detailed study of nonclassical examples (in particular, of the "Meixner analysis"), are natural and relevant.

In this paper we review some results of the "biorthogonal analysis" and of the analysis associated with the generalized Meixner measure [108]. In particular, from a general point of view, we consider stochastic integrals, stochastic derivatives, elements of a Wick calculus, and related topics. Note that these objects were not considered in details in the existing surveys [22-24]. As for the recent survey [80], the investigation of in that paper only stochastic integrals in a very specific context is presented there.

The present paper is organized in the following manner. In the first section, we deal with the "biorthogonal analysis". Namely, we introduce so-called generalized Appell-like polynomials (these polynomials form orthogonal bases in test function spaces), construct test function spaces and describe their properties, introduce pseudodifferential operators on the test function spaces and consider some properties of these operators, introduce a probability measure $\mu$ that satisfies certain conditions and consider generalized function spaces that are dual spaces of the test function spaces with respect to $L^{2}(\mu)$, derscribe natural orthogonal bases in the generalized function spaces; consider elements of a Wick calculus (an $S$-transform, a Wick multiplication, Wick versions of holomorphic functions) on the generalized function spaces; introduce and study an analog of the extended stochastic integral on the generalized function spaces, in particular, consider the interconnection between the "extended stochastic integration" and the Wick calculus, consider an example of an equation with Wick-type nonlinearities; introduce and study operators of stochastic differentiation on the generalized function spaces.

The second section is devoted to an analysis that is associated with the generalized Meixner measure $\mu$ (more exactly, in this section, we observe results that are associated with peculiarities of $\mu$ and can not be obtained from the general "biorthogonal theory", and describe the interconnection between these results and results of the "biorthogonal analysis"). Namely, following [108], we give a definition of the generalized Meixner measure, consider the space $L^{2}(\mu)$ and construct a natural orthogonal basis in this space; consider a so-called nonregular rigging of $L^{2}(\mu)$ (this rigging is the main "chain of spaces" in the "biorthogonal analysis"), describe natural orthogonal bases in spaces of this rigging and consider the interconnection between these bases and the bases that are constructed in the framework of the "biorthogonal analysis", introduce a socalled parametrized regular rigging of $L^{2}(\mu)$ and describe natural orthogonal bases 
in spaces of this rigging, consider elements of a Wick calculus on the parametrized generalized function spaces, introduce and study the extended stochastic integral with respect to the so-called Meixner process on the parametrized generalized function spaces (in particular, on the space of square integrable with respect to $\mu$ functions), consider the interconnection between the extended stochastic integration and the Wick calculus, consider an example of an equation with Wick-type nonlinearities, introduce and study stochastic derivatives and operators of stochastic differentiation on the parametrized generalized function spaces.

1. On a biorthogonal approach to construction of a non-Gaussian infinitedimensional analysis. 1.1. Generalized Appell-like polynomials. Let $\mathbb{R}_{+}:=[0,+\infty)$, $T:=\left\{\tau=\left(\tau_{1}, \tau_{2}\right)\right\}$, where $\tau_{1} \in \mathbb{N}, \tau_{2}: \mathbb{R}_{+} \rightarrow[1,+\infty)$ are infinite differentiable functions; $\left\{\mathcal{H}_{\tau}\right\}_{\tau \in T}$ are the Sobolev spaces on $\mathbb{R}_{+}$of order $\tau_{1}$ weighted by $\tau_{2}$, i.e., the scalar product in $\mathcal{H}_{\tau}$ has the form

$$
(f, g)_{\mathcal{H}_{\tau}}=\int_{\mathbb{R}_{+}}\left(f(u) g(u)+\sum_{k=1}^{\tau_{1}} f^{(k)}(u) g^{(k)}(u)\right) \tau_{2}(u) d u .
$$

We consider the chain

$$
\mathcal{D}^{\prime}=\operatorname{ind}_{\tau^{\prime} \in T} \lim _{-\tau^{\prime}} \supset \mathcal{H}_{-\tau} \supset \mathcal{H}:=L^{2}\left(\mathbb{R}_{+}, d u\right) \supset \mathcal{H}_{\tau} \supset \mathcal{D}:=\underset{\tau^{\prime} \in T}{\operatorname{pr} \lim } \mathcal{H}_{\tau^{\prime}},
$$

where $L^{2}\left(\mathbb{R}_{+}, d u\right)$ is the space of real-valued functions on $\mathbb{R}_{+}$square integrable with respect to the Lebesgue measuse, pr lim and ind lim denote projective and inductive limits with the coresponding topologies, respectively (e.g., [17]), $\mathcal{H}_{-\tau}$ and $\mathcal{D}^{\prime}$ are the spaces dual of $\mathcal{H}_{\tau}$ and $\mathcal{D}$ with respect to the "zero space" $\mathcal{H}$, respectively. One can show (e.g., as in [25]) that $\mathcal{D}$ is the Schwartz space of infinite-differentiable functions on $\mathbb{R}_{+}$ with compact supports.

Denote by the subindex $\mathbb{C}$ complexifications of spaces, by $\operatorname{Hol}_{0}\left(\mathcal{D}_{\mathbb{C}}\right)$ and $H_{o l}\left(\mathcal{D}_{\mathbb{C}}, \mathcal{D}_{\mathbb{C}}\right)$ algebras of (germs of) functions on $\mathcal{D}_{\mathbb{C}}$ holomorphic for $0 \in \mathcal{D}_{\mathbb{C}}$ with values in $\mathbb{C}$ and $\mathcal{D}_{\mathbb{C}}$ respectively (see, e.g., [35] for more details), by $\widehat{\otimes}$ a symmetric tensor product. Together with chain (1.1), we consider the chains

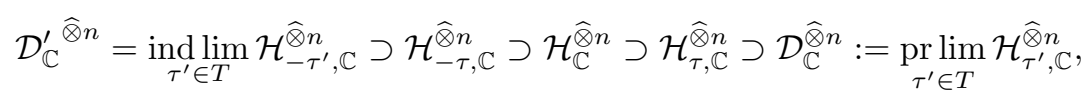

$n \in \mathbb{N}, \mathcal{D}_{\mathbb{C}}^{\prime \widehat{\otimes} 0}=\mathcal{H}_{-\tau, \mathbb{C}}^{\widehat{\otimes} 0}=\mathcal{H}_{\mathbb{C}}^{\widehat{\otimes} 0}=\mathcal{H}_{\tau, \mathbb{C}}^{\widehat{\otimes} 0}=\mathcal{D}_{\mathbb{C}}^{\widehat{\otimes} 0}:=\mathbb{C}$. Denote the norms in the spaces $\mathcal{H}_{-\tau, \mathbb{C}}^{\widehat{\otimes} n}, \mathcal{H}_{\mathbb{C}}^{\widehat{\otimes} n}$ and $\mathcal{H}_{\tau, \mathbb{C}}^{\widehat{\otimes} n}$ by $|\cdot|_{-\tau},|\cdot|_{0}$ and $|\cdot|_{\tau}$, respectively, the dual pairings between elements of negative and positive spaces of chains (1.2) by $\langle\cdot, \cdot\rangle$; these pairings are generated by the scalar products in $\mathcal{H}_{\mathbb{C}}^{\widehat{\otimes} n}$.

Assume that $\gamma \in \operatorname{Hol}_{0}\left(\mathcal{D}_{\mathbb{C}}\right), \gamma(0)=1, \alpha \in \operatorname{Hol}_{0}\left(\mathcal{D}_{\mathbb{C}}, \mathcal{D}_{\mathbb{C}}\right), \alpha(0)=0$. Let there exist the function $\alpha^{-1} \in \operatorname{Hol}_{0}\left(\mathcal{D}_{\mathbb{C}}, \mathcal{D}_{\mathbb{C}}\right)$ inverse to $\alpha$ and let $\chi: \mathbb{C} \rightarrow \mathbb{C}$ be an entire function such that $\chi(0)=1$ and in the decomposition

$$
\chi(u)=\sum_{n=0}^{\infty} \frac{\chi_{n}}{n !} u^{n},
$$

$\chi_{n} \neq 0$ for all $n \in \mathbb{Z}_{+}$. We consider a function $\chi^{\gamma, \alpha}(\lambda ; z):=\gamma(\lambda) \chi(\langle z, \alpha(\lambda)\rangle)$. It is easy to see that $\chi^{\gamma, \alpha}(\cdot ; z) \in \operatorname{Hol}_{0}\left(\mathcal{D}_{\mathbb{C}}\right)$ for each $z \in \mathcal{D}_{\mathbb{C}}^{\prime}$, therefore, using the Cauchy 
inequality (e.g., [35]) and the kernel theorem (e.g., [17]), one can show, that for $z \in \mathcal{D}_{\mathbb{C}}^{\prime}$ and $\lambda$ from some (depending on $z$ ) neighborhood of $0 \in \mathcal{D}_{\mathbb{C}}$, we have

$$
\chi^{\gamma, \alpha}(\lambda ; z)=\sum_{n=0}^{\infty} \frac{1}{n !}\left\langle P_{n}^{\chi, \gamma, \alpha}(z), \lambda^{\otimes n}\right\rangle
$$

where $P_{n}^{\alpha, \gamma, \alpha}(z) \in \mathcal{D}_{\mathbb{C}}^{\prime \widehat{\otimes} n}$ (moreover, if $z \in \mathcal{H}_{-\tau, \mathbb{C}}$, then $P_{n}^{\chi, \gamma, \alpha}(z) \in \mathcal{H}_{-\tau, \mathbb{C}}^{\widehat{\otimes} n}$ ). The polynomials $\left\{\left\langle P_{n}^{\chi, \gamma, \alpha}(z), f^{(n)}\right\rangle, f^{(n)} \in \mathcal{D}_{\mathbb{C}}^{\widehat{\otimes} n}, n \in \mathbb{Z}_{+}\right\}$are called generalized Appelllike polynomials (or Schefer polynomials in another terminology). The interested reader can find more detailed information about generalized Appell-like polynomials in, e.g., $[9,26]$ (one-dimensional case), [67, 77, 88, 91] (infinite-dimensional case).

1.2. Test function spaces. Let

$$
\mathcal{P}\left(\mathcal{D}^{\prime}\right)=\left\{\sum_{n=0}^{N}\left\langle x^{\otimes n}, g^{(n)}\right\rangle: x \in \mathcal{D}^{\prime}, g^{(n)} \in \mathcal{D}_{\mathbb{C}}^{\widehat{\otimes} n}, N \in \mathbb{Z}_{+}\right\}
$$

be the set of continuous polynomials on $\mathcal{D}^{\prime}$. One can show (e.g., [67]) that it is possible to understand $\mathcal{P}\left(\mathcal{D}^{\prime}\right)$ as the set of polynomials

$$
f(x)=\sum_{n=0}^{N_{f}}\left\langle P_{n}^{\chi, \gamma, \alpha}(x), f^{(n)}\right\rangle, \quad x \in \mathcal{D}^{\prime}, \quad f^{(n)} \in \mathcal{D}_{\mathbb{C}}^{\widehat{\otimes} n}, \quad N_{f} \in \mathbb{Z}_{+} .
$$

Let us introduce the family of Hilbert norms $\|\cdot\|_{\tau, q, \chi, \gamma, \alpha}, \tau \in T, q \in \mathbb{Z}_{+}$, on $\mathcal{P}\left(\mathcal{D}^{\prime}\right)$ by setting, for $f$ of form (1.4), the norm

$$
\|f\|_{\tau, q, \chi, \gamma, \alpha}^{2}:=\sum_{n=0}^{N_{f}}(n !)^{2} 2^{q n}\left|f^{(n)}\right|_{\tau}^{2} .
$$

By $\left(\mathcal{H}_{\tau}\right)_{q, \chi, \gamma, \alpha}$ denote a Hilbert space that is the closure of $\mathcal{P}\left(\mathcal{D}^{\prime}\right)$ with respect to norm (1.5). Let also $\left(\mathcal{H}_{\tau}\right)_{\chi, \gamma, \alpha}:=\operatorname{pr} \lim _{q \in \mathbb{Z}_{+}}\left(\mathcal{H}_{\tau}\right)_{q, \chi, \gamma, \alpha}, \quad(\mathcal{D})_{\chi, \gamma, \alpha}:=$ $:=\operatorname{pr} \lim _{\tau \in T, q \in \mathbb{Z}_{+}}\left(\mathcal{H}_{\tau}\right)_{q, \chi, \gamma, \alpha}$. The spaces $\left(\mathcal{H}_{\tau}\right)_{q, \chi, \gamma, \alpha},\left(\mathcal{H}_{\tau}\right)_{\chi, \gamma, \alpha},(\mathcal{D})_{\chi, \gamma, \alpha}$ are called Kondratiev test function spaces. It is easy to see that $f \in\left(\mathcal{H}_{\tau}\right)_{q, \chi, \gamma, \alpha}$ if and only if $f$ can be presented in the form

$$
f=\sum_{n=0}^{\infty}\left\langle P_{n}^{\chi, \gamma, \alpha}, f^{(n)}\right\rangle, \quad f^{(n)} \in \mathcal{H}_{\tau, \mathbb{C}}^{\widehat{\otimes} n}
$$

with

$$
\|f\|_{\left(\mathcal{H}_{\tau}\right)_{q, \chi, \gamma, \alpha}}^{2}=\sum_{n=0}^{\infty}(n !)^{2} 2^{q n}\left|f^{(n)}\right|_{\tau}^{2}<\infty .
$$

The interested reader can find a more detailed information about Kondratiev spaces in, e.g., $[7,12,22-24,67,68,88,90,91,112]$; here, we note only that

1) for $q \in \mathbb{Z}_{+}$sufficiently large, $\left(\mathcal{H}_{\tau}\right)_{q, \chi, \gamma, \alpha}$ are functional spaces, their elements are restrictions on $\mathcal{H}_{-\tau}$ of entire functions on $\mathcal{H}_{-\tau, \mathbb{C}}$, and for each function $f \in\left(\mathcal{H}_{\tau}\right)_{q, \chi, \gamma, \alpha}$ decomposition (1.6) is unique;

2) the spaces $\left(\mathcal{H}_{\tau}\right)_{\chi, \gamma, \alpha}$ and $(\mathcal{D})_{\chi, \gamma, \alpha}$ do not depend on $\gamma$ and $\alpha$, therefore below we denote these spaces by $\left(\mathcal{H}_{\tau}\right)_{\chi}$ and $(\mathcal{D})_{\chi}$, respectively. 
1.3. Pseudodifferential operators. Let $\nu \in \operatorname{Hol}_{0}\left(\mathcal{D}_{\mathbb{C}}\right)$. On $\mathcal{P}\left(\mathcal{D}^{\prime}\right)$, we define a pseudodifferential operator $\nu\left(D_{\chi}\right):=\sum_{n=0}^{\infty} \frac{1}{n !}\left\langle\nu_{n}, D_{\chi}^{\otimes n}\right\rangle$, where $\nu_{n} \in \mathcal{D}_{\mathbb{C}}^{\prime}{ }^{\widehat{\otimes} n}, n \in$ $\mathbb{Z}_{+}$, are the kernels from the decomposition $\nu(\lambda)=\sum_{n=0}^{\infty} \frac{1}{n !}\left\langle\nu_{n}, \lambda^{\otimes n}\right\rangle ;$

$$
\left\langle\nu_{n}, D_{\chi}^{\otimes n}\right\rangle\left\langle x^{\otimes m}, f^{(m)}\right\rangle:=1_{\{m \geq n\}} \frac{m ! \chi_{m-n}}{(m-n) ! \chi_{m}}\left\langle x^{\otimes m-n} \widehat{\otimes} \nu_{n}, f^{(m)}\right\rangle,
$$

where $\chi_{n} \in \mathbb{C}\left(n \in \mathbb{Z}_{+}\right)$are the coefficients from decomposition (1.3) for $\chi$; here and below, $1_{A}$ is the indicator of an event $A$. One can show $[67,77]$ that

1) for each $\nu_{n} \in \mathcal{D}_{\mathbb{C}}^{\prime} \widehat{\otimes}$,

$$
\left\langle\nu_{n}, \alpha^{-1}\left(D_{\chi}\right)^{\otimes n}\right\rangle\left\langle P_{m}^{\chi, \gamma, \alpha}(x), f^{(m)}\right\rangle=1_{\{m \geq n\}} \frac{m !}{(m-n) !}\left\langle P_{m-n}^{\chi, \gamma, \alpha}(x) \widehat{\otimes} \nu_{n}, f^{(m)}\right\rangle ;
$$

2) $\nu\left(\alpha^{-1}\left(D_{\chi}\right)\right)$ can be continued to a linear isometric operator acting from $\left(\mathcal{H}_{\tau}\right)_{q, \chi, \gamma, \alpha}$ to $\left(\mathcal{H}_{\tau}\right)_{q, \chi, \gamma \nu, \alpha}$ : for $f \in\left(\mathcal{H}_{\tau}\right)_{q, \chi, \gamma, \alpha}$ of form (1.6), we have

$$
\nu\left(\alpha^{-1}\left(D_{\chi}\right)\right) f=\sum_{n=0}^{\infty}\left\langle P_{n}^{\chi, \gamma \nu, \alpha}, f^{(n)}\right\rangle .
$$

1.4. Generalized function spaces. Let $\mu$ be a probability measure on $\left(\mathcal{D}^{\prime}, \mathcal{F}\left(\mathcal{D}^{\prime}\right)\right)$, here and below, $\mathcal{F}$ denotes the $\sigma$-algebra generated by cylindrical sets. Denote $\left(L^{2}\right)_{\mu}:=$ $:=L^{2}\left(\mathcal{D}^{\prime}, \mu\right)$ the space of functions $F: \mathcal{D}^{\prime} \rightarrow \mathbb{C}$ square integrable with respect to $\mu$. Let $\|\cdot\|_{\mu}$ be the norm in this space. In addition, we accept that $\mu$ satisfies the following assumptions:

1) there exists $\tau \in T$ such that $\mu\left(\mathcal{H}_{-\tau}\right)=1$;

2) there exists $K>1$ such that, for each $n \in \mathbb{Z}_{+},\left|\chi_{n}\right|\left|\left\||\cdot|{ }_{-\tau}^{n}\right\|_{\mu} \leq n ! K^{n}\right.$;

3) the set $\mathcal{P}\left(\mathcal{D}^{\prime}\right)$ of continuous polynomials on $\mathcal{D}^{\prime}$ is dense in $\left(L^{2}\right)_{\mu}$;

4) there exists a nonempty open set $O \subseteq \mathcal{H}_{-\tau}$ such that $\mu$ is positive on nonempty open subsets of $O$.

Let $\chi, \gamma, \alpha$ and $\mu$ satisfy all conditions mentioned above. If we exclude from $T$ some indexes (see [73] for more details), then it is posiible to show [79] that, for $q \in \mathbb{Z}_{+}$sufficiently large, the spaces $\left(\mathcal{H}_{\tau}\right)_{q, \chi, \gamma, \alpha}$ are embedded in $\left(L^{2}\right)_{\mu}$, and these embeddings are dense and continuous. Moreover, this result holds true if, inconvenient for verification, condition 4 on $\mu$ is substituted by the following one:

$\left.4^{\prime}\right)$ the polynomials $\left\{\left\langle P_{n}^{\chi, \gamma, \alpha}, f^{(n)}\right\rangle\right\}$ are orthogonal in $\left(L^{2}\right)_{\mu}$ and if $\|\left\langle P_{n}^{\chi, \gamma, \alpha}\right.$, $\left.f^{(n)}\right\rangle \|_{\mu}=0$, then $\left|f^{(n)}\right|_{\tau}=0$.

We note also that if $\chi=\exp$, then condition 2 is equivalent to holomorphy at zero of the Laplace transform of $\mu$ (e.g., [91]), and it follows from this holomorphy that condition 3 is satisfied (e.g., [109]).

We can now consider the chain

$$
(\mathcal{D})_{\chi, \mu}^{\prime} \supset\left(\mathcal{H}_{-\tau}\right)_{\chi, \mu} \supset\left(\mathcal{H}_{-\tau}\right)_{-q, \chi, \gamma, \alpha, \mu} \supset\left(L^{2}\right)_{\mu} \supset\left(\mathcal{H}_{\tau}\right)_{q, \chi, \gamma, \alpha} \supset\left(\mathcal{H}_{\tau}\right)_{\chi} \supset(\mathcal{D})_{\chi},
$$

where $\left(\mathcal{H}_{-\tau}\right)_{-q, \chi, \gamma, \alpha, \mu},\left(\mathcal{H}_{-\tau}\right)_{\chi, \mu}$ and $(\mathcal{D})_{\chi, \mu}^{\prime}$ are the spaces dual of $\left(\mathcal{H}_{\tau}\right)_{q, \chi, \gamma, \alpha},\left(\mathcal{H}_{\tau}\right)_{\chi}$ and $(\mathcal{D})_{\chi}$ with respect to $\left(L^{2}\right)_{\mu}$, correspondingly. It will be convenient to understand $\left(\mathcal{H}_{-\tau}\right)_{\chi, \mu}$ and $(\mathcal{D})_{\chi, \mu}^{\prime}$ as topological spaces with the inductive limit topologies. The negative spaces of chain (1.8) are called Kondratiev generalized functions spaces. 
Let us describe natural orthogonal bases in the spaces $\left(\mathcal{H}_{-\tau}\right)_{-q, \chi, \gamma, \alpha, \mu}$. Let $\langle\langle\cdot, \cdot\rangle\rangle_{\mu}$ denote the scalar product in $\left(L^{2}\right)_{\mu}$ (we preserve this notation for dual pairings generated by this scalar product); let

$$
\widetilde{\gamma}(\lambda):=\frac{1}{\langle\langle 1, \chi(\langle\cdot, \alpha(\lambda)\rangle)\rangle\rangle_{\mu}}
$$

One can show [67] that $\widetilde{\gamma} \in \operatorname{Hol}_{0}\left(\mathcal{D}_{\mathbb{C}}\right)$, therefore $\frac{\widetilde{\gamma}}{\gamma} \in \operatorname{Hol}_{0}\left(\mathcal{D}_{\mathbb{C}}\right)$ and one can consider the decomposition

$$
\frac{\widetilde{\gamma}(\lambda)}{\gamma(\lambda)}=\sum_{n=0}^{\infty} \frac{1}{n !}\left\langle\rho_{n}, \lambda^{\otimes n}\right\rangle, \quad \rho_{n} \in \mathcal{H}_{-\tau, \mathbb{C}}^{\widehat{\otimes} n}
$$

We set

$$
\begin{gathered}
Q_{\mu, m}^{\chi, \gamma, \alpha}\left(F^{(m)} ; \cdot\right):= \\
:=\sum_{k=m}^{\infty} \frac{1}{(k-m) !}\left(\left\langle F^{(m)} \widehat{\otimes} \rho_{k-m}, \alpha^{-1}\left(D_{\chi}\right)^{\otimes k}\right\rangle^{*} 1\right)(\cdot), \quad F^{(m)} \in \mathcal{H}_{-\tau, \mathbb{C}}^{\otimes},
\end{gathered}
$$

where $\left\langle F^{(m)} \widehat{\otimes} \rho_{k-m}, \alpha^{-1}\left(D_{\chi}\right)^{\otimes k}\right\rangle^{*}$ is the operator adjoint to pseudodifferential operator (1.7). It follows from results of [67] that the system of generalized functions $\left\{Q_{\mu, m}^{\chi, \gamma, \alpha}\left(F^{(m)}\right), F^{(m)} \in \mathcal{H}_{-\tau, \mathbb{C}}^{\widehat{\otimes} m}, m \in \mathbb{Z}_{+}\right\}$form orthogonal bases in $\left(\mathcal{H}_{-\tau}\right)_{-q, \chi, \gamma, \alpha, \mu}$ : $F \in\left(\mathcal{H}_{-\tau}\right)_{-q, \chi, \gamma, \alpha, \mu}$ if and only if there exists a sequence of kernels $\left\{F^{(m)} \in\right.$ $\left.\in \mathcal{H}_{-\tau, \mathbb{C}}^{\widehat{\otimes} m}\right\}_{m=0}^{\infty}$ such that

$$
F=\sum_{m=0}^{\infty} Q_{\mu, m}^{\chi, \gamma, \alpha}\left(F^{(m)}\right)
$$

with

$$
\|F\|_{-\tau,-q, \chi, \gamma, \alpha}^{2}:=\|F\|_{\left(\mathcal{H}_{-\tau}\right)_{-q, \chi, \gamma, \alpha, \mu}}^{2}=\sum_{m=0}^{\infty} 2^{-q m}\left|F^{(m)}\right|_{-\tau}^{2}<\infty .
$$

Moreover, the biorthogonality relation

$$
\left\langle\left\langle Q_{\mu, m}^{\chi, \gamma, \alpha}\left(F^{(m)}\right),\left\langle P_{n}^{\chi, \gamma, \alpha}, f^{(n)}\right\rangle\right\rangle\right\rangle_{\mu}=\delta_{m n} n !\left\langle F^{(n)}, f^{(n)}\right\rangle
$$

is satisfied, here, $\delta_{m n}$ is the Kroneker symbol.

Another (based on properties of Fock spaces) approach to the construction of orthogonal bases in generalized function spaces is described in [73].

1.5. Elements of a Wick calculus. Now we consider elements of a so-called Wick calculus on the generalized function spaces. For $F \in(\mathcal{D})_{\chi, \mu}^{\prime}$, we introduce a so-called $S$-transform by setting

$$
\left(S_{\chi, \gamma, \alpha, \mu} F\right)(\lambda):=\left\langle\left\langle F, \chi^{\gamma, \alpha}(\lambda ; \cdot)\right\rangle\right\rangle_{\mu}=\sum_{m=0}^{\infty}\left\langle F^{(m)}, \lambda^{\otimes m}\right\rangle,
$$

where $F^{(m)} \in \mathcal{D}_{\mathbb{C}}^{\prime} \widehat{\otimes} m, m \in \mathbb{Z}_{+}$, are the kernels from decomposition (1.10) for $F$; $\lambda \in \mathcal{D}_{\mathbb{C}}$. It is easy to show that the series in the right-hand side of (1.12) converges on 
some (depending on $F$ ) neighborhood of $0 \in \mathcal{D}_{\mathbb{C}}$. Further, one can show [67] that the $S$-transform is a one-to-one mapping between $(\mathcal{D})_{\chi, \mu}^{\prime}$ and $\operatorname{Hol}_{0}\left(\mathcal{D}_{\mathbb{C}}\right)$.

For $F, G \in(\mathcal{D})_{\chi, \mu}^{\prime}$ and a function $h: \mathbb{C} \rightarrow \mathbb{C}$, holomorphic for $\left(S_{\chi, \gamma, \alpha, \mu} F\right)(0)=$ $F^{(0)}$ (see (1.10)) we define a Wick product $F \diamond_{\mu}^{\chi, \gamma, \alpha} G \in(\mathcal{D})_{\chi, \mu}^{\prime}$ and a Wick version $h^{\diamond_{\mu}^{\chi, \gamma, \alpha}}(F) \in(\mathcal{D})_{\chi, \mu}^{\prime}$ by setting

$$
\begin{gathered}
F \diamond_{\mu}^{\chi, \gamma, \alpha} G:=S_{\chi, \gamma, \alpha, \mu}^{-1}\left(S_{\chi, \gamma, \alpha, \mu} F \cdot S_{\chi, \gamma, \alpha, \mu} G\right), \\
h^{\diamond_{\mu}^{\chi, \gamma, \alpha}}(F):=S_{\chi, \gamma, \alpha, \mu}^{-1} h\left(S_{\chi, \gamma, \alpha, \mu} F\right) .
\end{gathered}
$$

Note that if $\gamma(\lambda)$ has form (1.9), then $S_{\chi, \gamma, \alpha, \mu} 1 \equiv 1$, and therefore,

$$
h^{\diamond \underset{\mu}{\chi, \gamma, \alpha}}(F)=\sum_{n=0}^{\infty} h_{n}\left(F-F^{(0)}\right)^{\diamond_{\mu}^{\chi, \gamma, \alpha} n},
$$

where $F^{\diamond_{\mu}^{\chi, \gamma, \alpha} n}:=\underbrace{F \diamond_{\mu}^{\chi, \gamma, \alpha} \cdots \diamond_{\mu}^{\chi, \gamma, \alpha} F}_{n}=S_{\chi, \gamma, \alpha, \mu}^{-1}\left[\left(S_{\chi, \gamma, \alpha, \mu} F\right)^{n}\right], F_{\mu}^{\diamond_{\mu}^{\chi, \gamma, \alpha} 0}:=1$; and the coefficients $h_{n}$ from the decomposition

$$
h(u)=\sum_{n=0}^{\infty} h_{n}\left(u-F^{(0)}\right)^{n}
$$

belong to $\mathbb{C}$.

Let us consider the "coordinate form" of the Wick product and Wick versions of holomorphic functions. It is easy to calculate with the use of (1.12) that, for $F, G \in$ $(\mathcal{D})_{\chi, \mu}^{\prime}$,

$$
F \diamond_{\mu}^{\chi, \gamma, \alpha} G=\sum_{m=0}^{\infty} Q_{\mu, m}^{\chi, \gamma, \alpha}\left(\sum_{k=0}^{m} F^{(k)} \widehat{\otimes} G^{(m-k)}\right),
$$

where $F^{(k)}, G^{(k)} \in \mathcal{D}_{\mathbb{C}}^{\prime \widehat{\otimes} k}$ are the kernels from decompositions (1.10) for $F$ and $G$. If we apply the induction to this formula, then we obtain

$$
F_{1} \diamond_{\mu}^{\chi, \gamma, \alpha} \ldots \diamond_{\mu}^{\chi, \gamma, \alpha} F_{n}=\sum_{m=0}^{\infty} Q_{\mu, m}^{\chi, \gamma, \alpha}\left(\sum_{\substack{k_{1}, \ldots, k_{n} \in \mathbb{Z}_{+}, k_{1}+\cdots+k_{n}=m}} F_{1}^{\left(k_{1}\right)} \widehat{\otimes} \ldots \widehat{\otimes} F_{n}^{\left(k_{n}\right)}\right),
$$

where $F_{1}, \ldots, F_{n} \in(\mathcal{D})_{\chi, \mu}^{\prime}, F_{1}^{(k)}, \ldots, F_{n}^{(k)} \in \mathcal{D}_{\mathbb{C}}^{\prime \widehat{\otimes} k}$ are the kernels from decompositions (1.10) for $F_{1}, \ldots, F_{n}$, correspondingly. Further, substituting (1.12) in (1.13) and applying $S_{\chi, \gamma, \alpha, \mu}^{-1}$, we obtain

$$
h^{\diamond_{\mu}^{\chi, \gamma, \alpha}}(F)=Q_{\mu, 0}^{\chi, \gamma, \alpha}\left(h_{0}\right)+\sum_{m=1}^{\infty} Q_{\mu, m}^{\chi, \gamma, \alpha}\left(\sum_{n=1}^{m} h_{n} \sum_{\substack{k_{1}, \ldots, k_{n} \in \mathbb{N}, k_{1}+\cdots+k_{n}=m}} F^{\left(k_{1}\right)} \widehat{\otimes} \ldots \widehat{\otimes} F^{\left(k_{n}\right)}\right) .
$$

In particular, note that,

$$
Q_{\mu, n}^{\chi, \gamma, \alpha}\left(F^{(n)}\right) \diamond_{\mu}^{\chi, \gamma, \alpha} Q_{\mu, m}^{\chi, \gamma, \alpha}\left(G^{(m)}\right)=Q_{\mu, n+m}^{\chi, \gamma, \alpha}\left(F^{(n)} \widehat{\otimes} G^{(m)}\right) .
$$

Consider the space $\left(\mathcal{H}_{-\tau}\right)_{\chi, \mu} \subset(\mathcal{D})_{\chi, \mu}^{\prime}$ instead of $(\mathcal{D})_{\chi, \mu}^{\prime}$. The following statement [70] is true. 
Theorem 1.1. Let $F, G \in\left(\mathcal{H}_{-\tau}\right)_{\chi, \mu}$ and $h: \mathbb{C} \rightarrow \mathbb{C}$ be a function holomorphic at $\left(S_{\chi, \gamma, \alpha, \mu} F\right)(0)$. Then $F \diamond_{\mu}^{\chi, \gamma, \alpha} G \in\left(\mathcal{H}_{-\tau}\right)_{\chi, \mu}, h^{\diamond{ }_{\mu}^{\chi, \gamma, \alpha}}(F) \in\left(\mathcal{H}_{-\tau}\right)_{\chi, \mu}$, and coordinate formulas (1.14)-(1.16) hold true. Moreover, the Wick product is continuous in the topology of $\left(\mathcal{H}_{-\tau}\right)_{\chi, \mu}$ : for any $F_{1}, \ldots, F_{n} \in\left(\mathcal{H}_{-\tau}\right)_{\chi, \mu}$,

$$
\begin{gathered}
\left\|F_{1} \diamond_{\mu}^{\chi, \gamma, \alpha} \ldots \diamond_{\mu}^{\chi, \gamma, \alpha} F_{n}\right\|_{-\tau,-q, \chi, \gamma, \alpha} \leq \\
\leq c(n-1)\left\|F_{1}\right\|_{-\tau,-(q-1), \chi, \gamma, \alpha} \ldots\left\|F_{n}\right\|_{-\tau,-(q-1), \chi, \gamma, \alpha}
\end{gathered}
$$

$(\operatorname{see}(1.11))$, where $c(n):=\sqrt{\max _{m \in \mathbb{Z}_{+}}\left[2^{-m}(m+1)^{n}\right]} ; q \in \mathbb{N}$ is such that $F_{1}, \ldots, F_{n} \in$ $\left(\mathcal{H}_{-\tau}\right)_{-(q-1), \chi, \gamma, \alpha, \mu}$.

1.6. An analog of the extended stochastic integral. Let us consider now an analog of the extended (Skorohod-type, see [57, 63, 64, 110]) stochastic integral in the "biorthogonal analysis" (the reader can find a more detailed presentation in [74], see also [73]). In order to explain the idea of our construction, first we briefly recall the construction of the stochastic integral in the Gaussian analysis.

Let $\mu$ be the standard Gaussian measure on $\left(\mathcal{D}^{\prime}, \mathcal{F}\left(\mathcal{D}^{\prime}\right)\right)$, i.e., a probability measure with the Laplace transform

$$
l_{\mu}(\lambda)=\int_{\mathcal{D}^{\prime}} e^{\langle x, \lambda\rangle} \mu(d x)=e^{\langle\lambda, \lambda\rangle / 2} .
$$

As is well known (e.g., [54]), one can consider as an orthogonal basis in $\left(L^{2}\right)_{\mu}$ $\left(\left(L^{2}\right)_{\mu}\right.$-limits of $)$ the generalized Hermite polynomials $\left\langle H_{n}, F^{(n)}\right\rangle$; these polynomials are generalized Appell-like polynomials with $\chi=\exp , \gamma(\lambda)=\exp \left\{-\frac{1}{2}\langle\lambda, \lambda\rangle\right\}$, $\alpha(\lambda)=\lambda$.

Let now $F \in\left(L^{2}\right)_{\mu} \otimes \mathcal{H}_{\mathbb{C}}$. Then $F$ can be presented in the form

$$
F(\cdot)=\sum_{n=0}^{\infty}\left\langle H_{n}, F^{(n)}\right\rangle, \quad F^{(n)} \in \mathcal{H}_{\mathbb{C}}^{\widehat{\otimes} n} \otimes \mathcal{H}_{\mathbb{C}} ;
$$

and if, in addition, $F$ is integrable in the extended (Skorohod) sense, i.e.,

$$
\sum_{n=0}^{\infty}(n+1) !\left|\widehat{F}^{(n)}\right|_{0}^{2}<\infty
$$

where, for each $n \in \mathbb{Z}_{+}, \widehat{F}^{(n)} \in \mathcal{H}_{\mathbb{C}}^{\widehat{\otimes} n+1}$ is the projection of $F^{(n)}$ onto $\mathcal{H}_{\mathbb{C}}^{\widehat{\otimes} n+1}$, then the extended stochastic integral of $F$ with respect to a Wiener process $W$ has the form (e.g., $[56])$

$$
\int_{0}^{\infty} F(u) \widehat{d} W_{u}=\sum_{n=0}^{\infty}\left\langle H_{n+1}, \widehat{F}^{(n)}\right\rangle
$$

Remark. The described construction of the extended stochastic integral is not unique. For example, it was shown in [31] that (in the Gaussian case) the extended stochastic integral can be interpreted as the logarithmic derivative of the Gaussian measure in the line of a vector field. By analogy, i.e., using an integration by parts formula, one can 
construct stochastic integrals in non-Gaussian cases, see, e.g., [10, 99, 100, 107]. Note that, for groups of nonlinear transformations, the definitions of the logarithmic derivative and of the extended stochastic integral were given in [111]. A very general construction of (the analog of) the extended stochastic integral (that is based on a formal differential rule and on the corresponding integration by parts formula) is presented in [47], see also [36-46, 48-50].

Return now to the "biorthogonal analysis". Let $F \in(\mathcal{D})_{\chi, \mu}^{\prime} \otimes \mathcal{H}_{\mathbb{C}}$. Then

$$
F(\cdot)=\sum_{m=0}^{\infty} Q_{\mu, m}^{\chi, \gamma, \alpha}\left(F^{(m)}\right), \quad F_{\cdot}^{(m)} \in \mathcal{D}_{\mathbb{C}}^{\prime \widehat{\otimes} m} \otimes \mathcal{H}_{\mathbb{C}}
$$

By analogy with (1.17), for any $t_{1}, t_{2} \in[0,+\infty], t_{1}<t_{2}$, one can define an analog of the extended stochastic integral by setting

$$
\mathbf{I}_{\left[t_{1}, t_{2}\right)}^{\chi, \gamma, \alpha, \mu}(F):=\sum_{m=0}^{\infty} Q_{\mu, m+1}^{\chi, \gamma, \alpha}\left(\widehat{F}_{\left[t_{1}, t_{2}\right)}^{(m)}\right) \in(\mathcal{D})_{\chi, \mu}^{\prime},
$$

where $\widehat{F}_{\left[t_{1}, t_{2}\right)}^{(m)} \in \mathcal{D}_{\mathbb{C}}^{\prime \widehat{\otimes} m+1}\left(m \in \mathbb{Z}_{+}\right)$are the projections of $F^{(m)} 1_{\left[t_{1}, t_{2}\right)}(\cdot)$ onto $\mathcal{D}_{\mathbb{C}}^{\prime}{ }^{\widehat{\otimes} m+1}$. It is easy to show [74] that, for all $t_{1}, t_{2} \in[0,+\infty], t_{1}<t_{2}, \mathbf{I}_{\left[t_{1}, t_{2}\right)}^{\chi, \gamma, \alpha, \mu}:(\mathcal{D})_{\chi, \mu}^{\prime} \otimes$ $\mathcal{H}_{\mathbb{C}} \rightarrow(\mathcal{D})_{\chi, \mu}^{\prime}$ is a linear continuous operator.

The forthcoming statement follows from results of [74].

Theorem. For all $t_{1}, t_{2} \in[0,+\infty], t_{1}<t_{2}$, and $F \in(\mathcal{D})_{\chi, \mu}^{\prime} \otimes \mathcal{H}_{\mathbb{C}}$, the relation

$$
\mathbf{I}_{\left[t_{1}, t_{2}\right)}^{\chi, \gamma, \alpha}(F)=\int_{t_{1}}^{t_{2}} F(u) \diamond_{\mu}^{\chi, \gamma, \alpha} Q_{\mu, 1}^{\chi, \gamma, \alpha}\left(\delta_{u}\right) d u
$$

is satisfied, where $\delta_{u}$ is the Dirac delta-function, and the integral on the right-hand side is a Pettis integral.

By analogy with the classical Gaussian analysis, as an example, we consider a simple “integral equation with a Wick-type nonlinearity". Let

$$
X_{t}=X_{0}+r \int_{0}^{t} X_{u} \diamond_{\mu}^{\chi, \gamma, \alpha}\left(N-X_{u}\right) d u+v \mathbf{I}_{[0, t)}^{\chi, \gamma, \alpha, \mu}\left(X . \diamond_{\mu}^{\chi, \gamma, \alpha}(N-X .)\right),
$$

where $X_{0} \in(\mathcal{D})_{\chi, \mu}^{\prime}$ (correspondingly $\left.\left(\mathcal{H}_{-\tau}\right)_{\chi, \mu}\right), N, r, v \in \mathbb{R}, N>0, r>0$, $\left(S_{\chi, \gamma, \alpha, \mu} X_{0}\right)(0)>0$. Note that, in the Gaussian analysis, (1.18) is the so-called "population growth equation" (see, e.g., [94]). Applying to (1.18) the $S$-transform, solving the obtained equation and applying the inverse $S$-transform, we obtain the solution

$$
\begin{gathered}
X_{t}=N\left[S_{\chi, \gamma, \alpha, \mu}^{-1} 1+\left(N X_{0}^{\diamond_{\mu}^{\chi, \gamma, \alpha}(-1)}-S_{\chi, \gamma, \alpha, \mu}^{-1} 1\right)\right. \\
\diamond_{\mu}^{\chi, \gamma, \alpha} \exp ^{\diamond_{\mu}^{\chi, \gamma, \alpha}}\left\{-N\left(r t S_{\chi, \gamma, \alpha, \mu}^{-1} 1+v Q_{\mu, 1}^{\chi, \gamma, \alpha}\left(1_{[0, t)}\right)\right\}\right]^{\diamond_{\mu}^{\chi, \gamma, \alpha}(-1)} \in(\mathcal{D})_{\chi, \mu}^{\prime}
\end{gathered}
$$

(correspondingly $\left(\mathcal{H}_{-\tau}\right)_{\chi, \mu}$ ), here $Y^{\diamond_{\mu}^{\chi, \gamma, \alpha}(-1)}:=S_{\chi, \gamma, \alpha, \mu}^{-1}\left(\frac{1}{S_{\chi, \gamma, \alpha, \mu} Y}\right)$. 
As is well known, in the Gaussian analysis, the extended stochastic integral is the adjoint operator to the stochastic derivative. In the "biorthogonal analysis", the situation is quite similar; let us explain this in details. We define a stochastic derivative $\partial^{\chi, \alpha}:(\mathcal{D})_{\chi} \rightarrow(\mathcal{D})_{\chi} \otimes \mathcal{H}_{\mathbb{C}}$ by setting, for $f \in(\mathcal{D})_{\chi}$,

$$
\partial^{\chi, \alpha} f:=\left\langle\delta ., \alpha^{-1}\left(D_{\chi}\right)\right\rangle f=\sum_{n=0}^{\infty}(n+1)\left\langle P_{n}^{\chi, \gamma, \alpha}, f^{(n+1)}(\cdot)\right\rangle
$$

(see (1.7)), where $f^{(n+1)}(\cdot) \in \mathcal{D}_{\mathbb{C}}^{\widehat{\otimes} n} \otimes \mathcal{H}_{\mathbb{C}}\left(n \in \mathbb{Z}_{+}\right)$are the kernels $f^{(n+1)} \in \mathcal{D}_{\mathbb{C}}^{\widehat{\otimes} n+1} \subset$ $\subset \mathcal{D}_{\mathbb{C}}^{\widehat{\otimes} n} \otimes \mathcal{H}_{\mathbb{C}}$ from decomposition (1.6) for $f$ (these kernels can be considered as elements of $\left.\mathcal{D}_{\mathbb{C}}^{\widehat{\otimes} n} \otimes \mathcal{H}_{\mathbb{C}}\right)$. By using the results of $[74,73]$, we obtain the following statements.

Theorem 1.2. For all $t_{1}, t_{2} \in[0,+\infty], t_{1}<t_{2}$, the operators $\mathbf{I}_{\left[t_{1}, t_{2}\right)}^{\chi, \gamma, \alpha, \mu}:(\mathcal{D})_{\chi, \mu}^{\prime} \otimes$ $\mathcal{H}_{\mathbb{C}} \rightarrow(\mathcal{D})_{\chi, \mu}^{\prime}$ and $1_{\left[t_{1}, t_{2}\right)}(\cdot) \partial^{\chi, \alpha}:(\mathcal{D})_{\chi} \rightarrow(\mathcal{D})_{\chi} \otimes \mathcal{H}_{\mathbb{C}}$ are adjoint one to another:

$$
\left(\mathbf{I}_{\left[t_{1}, t_{2}\right)}^{\chi, \gamma, \alpha, \mu}\right)^{*}=1_{\left[t_{1}, t_{2}\right)}(\cdot) \partial_{\cdot}^{\chi, \alpha} ; \quad\left(1_{\left[t_{1}, t_{2}\right)}(\cdot) \partial_{\cdot}^{\chi, \alpha}\right)^{*}=\mathbf{I}_{\left[t_{1}, t_{2}\right)}^{\chi, \gamma, \alpha, \mu}
$$

In particular, the operator $\mathbf{I}_{\left[t_{1}, t_{2}\right)}^{\chi, \gamma, \alpha, \mu}$ does not depend on $\gamma$ and can be denoted by $\mathbf{I}_{\left[t_{1}, t_{2}\right)}^{\chi, \alpha}$.

Corollary 1.1. The operator $\mathbf{I}_{\left[t_{1}, t_{2}\right)}^{\chi, \alpha, \mu}$ can be written in the form

$$
\mathbf{I}_{\left[t_{1}, t_{2}\right)}^{\chi, \alpha, \mu}(F)=\int_{t_{1}}^{t_{2}}\left(\partial_{u}^{\chi, \alpha}\right)^{\dagger} F(u) d u
$$

where $\left(\partial_{u}^{\chi, \alpha}\right)^{\dagger}:(\mathcal{D})_{\chi, \mu}^{\prime} \rightarrow(\mathcal{D})_{\chi, \mu}^{\prime}$ is the operator adjoint to $\partial_{u}^{\chi, \alpha}, u \in \mathbb{R}_{+} ;$the integral on the right-hand side is a Pettis integral.

Similar results are true if we consider operators $\mathbf{I}_{\left[t_{1}, t_{2}\right)}^{\chi, \alpha, \mu}$ and $\partial^{\chi, \alpha}$ on "pre-limit" spaces.

1.7. Operators of stochastic differentiation. Unfortunatelly, the operator $\partial^{\chi, \alpha}$, generally speaking, cannot be continued on the generalized function spaces by a natural way. Nevertheless, one can define and study a natural analog of this operator; the reader can find a more detailed information in [72].

Let us now consider operators of stochastic differentiation on the generalized function spaces. Let $F^{(m)} \in \mathcal{D}_{\mathbb{C}}^{\prime \widehat{\otimes} m}, f^{(n)} \in \mathcal{D}_{\mathbb{C}}^{\widehat{\otimes} n}, m>n$. We define a "pairing" $\left\langle F^{(m)}\right.$, $\left.f^{(n)}\right\rangle \in \mathcal{D}_{\mathbb{C}}^{\prime \widehat{\otimes} m-n}$ by setting, for each $g^{(m-n)} \in \mathcal{D}_{\mathbb{C}}^{\widehat{\otimes} m-n}$, the relation

$$
\left\langle\left\langle F^{(m)}, f^{(n)}\right\rangle, g^{(m-n)}\right\rangle=\left\langle F^{(m)}, f^{(n)} \widehat{\otimes} g^{(m-n)}\right\rangle .
$$

Further, for arbitrary $f^{(n)} \in \mathcal{D}_{\mathbb{C}}^{\widehat{\otimes} n}, n \in \mathbb{Z}_{+}$, we define a linear operator $\left(\widetilde{\mathbb{D}}_{\chi, \gamma, \alpha, \mu}^{n}\right)\left(f^{(n)}\right)$ in $(\mathcal{D})_{\chi, \mu}^{\prime}$ by setting, for $F \in(\mathcal{D})_{\chi, \mu}^{\prime}$, the relation

$$
\left(\widetilde{\mathbb{D}}_{\chi, \gamma, \alpha, \mu}^{n} F\right)\left(f^{(n)}\right):=\sum_{m=0}^{\infty} \frac{(m+n) !}{m !} Q_{\mu, m}^{\chi, \gamma, \alpha}\left(\left\langle F^{(m+n)}, f^{(n)}\right\rangle\right),
$$

where $F^{(m)} \in \mathcal{D}_{\mathbb{C}}^{\prime \widehat{\otimes} m}, m \in \mathbb{Z}_{+}$, are the kernels from decomposition (1.10) for $F$. It follows from results of [72] that $\left(\widetilde{\mathbb{D}}_{\chi, \gamma, \alpha, \mu}^{n} \circ\right)\left(f^{(n)}\right)$ is a continuous operator in $(\mathcal{D})_{\chi, \mu}^{\prime}$, and the following statement is true. 
Theorem 1.3. The operator $\widetilde{\mathbb{D}}_{\chi, \gamma, \alpha, \mu}^{n}$ has the following properties: for $g_{1}^{(1)}, \ldots, g_{n}^{(1)} \in \mathcal{D}_{\mathbb{C}}$ and $F \in(\mathcal{D})_{\chi, \mu}^{\prime}$,

$$
\begin{aligned}
& \underbrace{\left(\widetilde{\mathbb{D}}_{\chi, \gamma, \alpha, \mu}^{1}\left(\ldots\left(\widetilde{\mathbb{D}}_{\chi, \gamma, \alpha, \mu}^{1}\left(\left(\widetilde{\mathbb{D}}_{\chi, \gamma, \alpha, \mu}^{1} F\right)\left(g_{1}^{(1)}\right)\right)\right)\left(g_{2}^{(1)}\right) \ldots\right)\right)\left(g_{n}^{(1)}\right)=}_{n} \\
& =\left(\widetilde{\mathbb{D}}_{\chi, \gamma, \alpha, \mu}^{n} F\right)\left(g_{1}^{(1)} \widehat{\otimes} \ldots \widehat{\otimes} g_{n}^{(1)}\right) ;
\end{aligned}
$$

for each $F \in(\mathcal{D})_{\chi, \mu}^{\prime}$, the kernels $F^{(m)} \in \mathcal{D}_{\mathbb{C}}^{\prime \widehat{\otimes} m}$ from decomposition (1.10) can be presented in the form

$$
F^{(m)}=\frac{1}{m !} \mathbf{E}\left(\widetilde{\mathbb{D}}_{\chi, \gamma, \alpha, \mu}^{m} F\right)
$$

i.e., for each $f^{(m)} \in \mathcal{D}_{\mathbb{C}}^{\widehat{\otimes} m}$, we have $\left\langle F^{(m)}, f^{(m)}\right\rangle=\frac{1}{m !} \mathbf{E}\left[\left(\widetilde{\mathbb{D}}_{\chi, \gamma, \alpha, \mu}^{m} F\right)\left(f^{(m)}\right)\right]$, where $\mathbf{E}$ denotes the expectation: $\mathbf{E} \circ=\langle\langle\circ, 1\rangle\rangle_{\mu}$;

the operator adjoint to $\widetilde{\mathbb{D}}_{\chi, \gamma, \alpha, \mu}^{n}$ has a form

$$
\left(\widetilde{\mathbb{D}}_{\chi, \gamma, \alpha, \mu}^{n} g\right)\left(f^{(n)}\right)^{*}=\sum_{k=0}^{\infty}\left\langle P_{k+n}^{\chi, \gamma, \alpha}, f^{(n)} \widehat{\otimes} g^{(k)}\right\rangle, \quad f^{(n)} \in \mathcal{D}_{\overparen{C}}^{\widehat{\otimes} n},
$$

where $g^{(k)} \in \mathcal{D}_{\mathbb{C}_{\sim}}^{\widehat{\otimes} k}, k \in \mathbb{Z}_{+}$, are the kernels from decomposition $(1.4)$ for $g \in(\mathcal{D})_{\chi}$;

the operator $\widetilde{\mathbb{D}}_{\chi, \gamma, \alpha, \mu}^{1}$ is a pre-image of the directional derivative of $S_{\chi, \gamma, \alpha, \mu} \circ$ under the $S$-transform, i.e., for any $F \in(\mathcal{D})_{\chi, \mu}^{\prime}$ and $g \in \mathcal{D}_{\mathbb{C}}$,

$$
\left(\widetilde{\mathbb{D}}_{\chi, \gamma, \alpha, \mu}^{1} F\right)(g)=S_{\chi, \gamma, \alpha, \mu}^{-1} D_{g}\left(S_{\chi, \gamma, \alpha, \mu} F\right),
$$

where $D_{g}$ is the directional derivative in the direction $g$;

the operator $\widetilde{\mathbb{D}}_{\chi, \gamma, \alpha, \mu}^{1}$ is a differentiation with respect to the Wick product, i.e., for all $F, G \in(\mathcal{D})_{\chi, \mu}^{\prime}$,

$$
\widetilde{\mathbb{D}}_{\chi, \gamma, \alpha, \mu}^{1}\left(F \diamond_{\mu}^{\chi, \gamma, \alpha} G\right)=\left(\widetilde{\mathbb{D}}_{\chi, \gamma, \alpha, \mu}^{1} F\right) \diamond_{\mu}^{\chi, \gamma, \alpha} G+F \diamond_{\mu}^{\chi, \gamma, \alpha}\left(\widetilde{\mathbb{D}}_{\chi, \gamma, \alpha, \mu}^{1} G\right)
$$

for any $n \in \mathbb{Z}_{+}, F \in(\mathcal{D})_{\chi, \mu}^{\prime}$, and a function $h: \mathbb{C} \rightarrow \mathbb{C}$ holomorphic for $\left(S_{\chi, \gamma, \alpha, \mu} F\right)(0)$ the ralations

$$
\begin{aligned}
& \widetilde{\mathbb{D}}_{\chi, \gamma, \alpha, \mu}^{1} F^{\diamond_{\mu}^{\chi, \gamma, \alpha} n}=n F^{\diamond_{\mu}^{\chi, \gamma, \alpha} n-1} \diamond_{\mu}^{\chi, \gamma, \alpha}\left(\widetilde{\mathbb{D}}_{\chi, \gamma, \alpha, \mu}^{1} F\right), \\
& \widetilde{\mathbb{D}}_{\chi, \gamma, \alpha, \mu}^{1} h^{\diamond_{\mu}^{\chi, \gamma, \alpha}}(F)=h^{\prime \diamond_{\mu}^{\chi, \gamma, \alpha}}(F) \diamond_{\mu}^{\chi, \gamma, \alpha}\left(\widetilde{\mathbb{D}}_{\chi, \gamma, \alpha, \mu}^{1} F\right)
\end{aligned}
$$

are satisfied; here, $h^{\prime}$ is the usual derivative of $h$;

for all $t_{1}, t_{2} \in[0,+\infty], t_{1}<t_{2}$, and $F \in(\mathcal{D})_{\chi, \mu}^{\prime} \otimes \mathcal{H}_{\mathbb{C}}$,

$$
\left(\widetilde{\mathbb{D}}_{\chi, \gamma, \alpha, \mu}^{1} \mathbf{I}_{\left[t_{1}, t_{2}\right)}^{\chi, \alpha, \mu}(F)\right)(\circ)=\mathbf{I}_{\left[t_{1}, t_{2}\right)}^{\chi, \alpha, \mu}\left(\left(\widetilde{\mathbb{D}}_{\chi, \gamma, \alpha, \mu}^{1} F\right)(\circ)\right)+\int_{t_{1}}^{t_{2}} F(u) \circ(u) d u,
$$

where the last integral on the right-hand side is a Pettis integral. 
An information about possible applications of the operators $\widetilde{\mathbb{D}}_{\chi, \gamma, \alpha, \mu}^{n}$ and the corresponding examples can be found in, e.g., [11, 72].

2. Elements of the white noise analysis associated with the generalized Meixner measure. 2.1. The generalized Meixner measure. First, we define the generalized Meixner measure (see [108] for more details and explanations). Let $\rho, \nu: \mathbb{R}_{+} \rightarrow \mathbb{C}$ be smooth functions such that

$$
\theta \stackrel{d f}{=} \rho-\nu: \mathbb{R}_{+} \rightarrow \mathbb{R}, \quad \eta \stackrel{d f}{=} \rho \nu: \mathbb{R}_{+} \rightarrow \mathbb{R}_{+}
$$

and, moreover, $\theta$ and $\eta$ are bounded on $\mathbb{R}_{+}$. Further, let, for each $u \in \mathbb{R}_{+}, v_{\rho(u), \nu(u)}(d s)$ be a probability measure on $(\mathbb{R}, \mathcal{B}(\mathbb{R}))$ (here $\mathcal{B}$ denotes the Borel $\sigma$-algebra) that is defined by its Fourier transform

$$
\begin{gathered}
\int_{\mathbb{R}} e^{i \lambda s} v_{\rho(u), \nu(u)}(d s)=\exp \{-i \lambda(\rho(u)+\nu(u))+ \\
\left.+2 \sum_{m=1}^{\infty} \frac{(\rho(u) \nu(u))^{m}}{m}\left[\sum_{n=2}^{\infty} \frac{(-i \lambda)^{n}}{n !}\left(\nu^{n-2}(u)+\nu^{n-3}(u) \rho(u)+\cdots+\rho^{n-2}(u)\right)\right]^{m}\right\} .
\end{gathered}
$$

A probability measure $\mu$ on the measurable space $\left(\mathcal{D}^{\prime}, \mathcal{F}\left(\mathcal{D}^{\prime}\right)\right)$ with the Fourier transform

$$
\int_{\mathcal{D}^{\prime}} e^{i\langle x, \xi\rangle} \mu(d x)=\exp \left\{\int_{\mathbb{R}_{+}} d u \int_{\mathbb{R}^{\prime}} v_{\rho(u), \nu(u)}(d s) \frac{1}{s^{2}}\left(e^{i s \xi(u)}-1-i s \xi(u)\right)\right\}
$$

is called the generalized Meixner measure.

Depending on parametrs $\rho$ and $\nu, \mu$ can be, in particular, the Gaussian, Poissonian, Pascal, Meixner or gamma measure.

It was proved in [108] that the generalized Meixner measure $\mu$ is the measure of a generalized random process (in the sense of [51]) with independent values; and the Laplace transform $l_{\mu}$ of $\mu$ is a holomorphic for zero function.

2.2. The square integrable function space. Let $\left(L^{2}\right)=L^{2}\left(\mathcal{D}^{\prime}, \mu\right)$ be the space of complex-valued square integrable with respect to the generalized Meixner measure $\mu$ functions on $\mathcal{D}^{\prime}$. Denote by $\langle\langle\cdot \cdot \cdot \cdot\rangle\rangle$ the scalar product in $\left(L^{2}\right)$, this notation will be preserved for generated by $\langle\langle\cdot, \cdot\rangle\rangle$ dual pairings. We construct now a natural orthogonal basis in $\left(L^{2}\right)$. For $n \in \mathbb{N}$ denote by $\overline{\mathcal{P}}_{n}$ the closure in $\left(L^{2}\right)$ of the set of all continuous polynomials on $\mathcal{D}^{\prime}$ of degree $\leq n, \overline{\mathcal{P}}_{0}:=\mathbb{C}$, let also $\left(L_{n}^{2}\right):=\overline{\mathcal{P}}_{n} \ominus \overline{\mathcal{P}}_{n-1}$-the orthogonal difference in $\left(L^{2}\right),\left(L_{0}^{2}\right):=\mathbb{C}$. Since $\mu$ has a holomorphic at zero Laplace transform, the set of continuous polynomials on $\mathcal{D}^{\prime}$ is dense in $\left(L^{2}\right)$ [109], therefore $\left(L^{2}\right)=\bigoplus_{n=0}^{\infty}\left(L_{n}^{2}\right)$. For each $f^{(n)} \in \mathcal{D}_{\mathbb{C}}^{\widehat{\otimes} n}, n \in \mathbb{Z}_{+}$, we define $:\left\langle x^{\otimes n}, f^{(n)}\right\rangle:$ as the orthogonal projection of $\left\langle x^{\otimes n}, f^{(n)}\right\rangle$ onto $\left(L_{n}^{2}\right)$. It follows from results of [108] that $:\left\langle x^{\otimes n}, f^{(n)}\right\rangle:=\left\langle P_{n}(x), f^{(n)}\right\rangle$, where $P_{n}(x) \in \mathcal{D}_{\mathbb{C}}^{\prime}{ }^{\widehat{\otimes} n}\left(n \in \mathbb{Z}_{+}\right)$are the kernels of generalized Appell-like polynomials with $\chi=\exp$, 


$$
\begin{gathered}
\alpha(\lambda)=\lambda+\sum_{n=2}^{\infty} \frac{\lambda^{n}}{n}\left(\rho^{n-1}+\rho^{n-2} \nu+\cdots+\nu^{n-1}\right), \\
\gamma(\lambda)=\frac{1}{l_{\mu}(\alpha(\lambda))} \\
=\exp \left\{-\int_{\mathbb{R}_{+}}\left(\frac{\lambda^{2}(u)}{2}+\sum_{n=3}^{\infty} \frac{\lambda^{n}(u)}{n}\left(\rho^{n-2}(u)+\rho^{n-3}(u) \nu(u)+\cdots+\nu^{n-2}(u)\right)\right) d u\right\} .
\end{gathered}
$$

We say that the polynomials $\left\{\left\langle P_{n}, f^{(n)}\right\rangle, f^{(n)} \in \mathcal{D}_{\mathbb{C}}^{\widehat{\otimes} n}, n \in \mathbb{Z}_{+}\right\}$are called the generalized Meixner polynomials.

Let us define a scalar product $\langle\cdot, \cdot\rangle_{\text {ext }}$ on $\mathcal{D}_{\mathbb{C}}^{\widehat{\otimes} n}, n \in \mathbb{Z}_{+}$, by setting for $f^{(n)}, g^{(n)} \in$ $\in \mathcal{D}_{\mathbb{C}}^{\widehat{\otimes} n}$

$$
\left\langle f^{(n)}, g^{(n)}\right\rangle_{\mathrm{ext}}:=\frac{1}{n !} \int_{\mathcal{D}^{\prime}}\left\langle P_{n}, f^{(n)}\right\rangle\left\langle P_{n}, g^{(n)}\right\rangle \mu(d x) .
$$

It follows from results of [108] that

$$
\begin{aligned}
& \left\langle f^{(n)}, g^{(n)}\right\rangle_{\mathrm{ext}}=\sum_{\substack{k, l_{j}, s_{j} \in \mathbb{N}: j_{j=1, \ldots, k}, l_{1}>l_{2}>\cdots>l_{k}, l_{1} s_{1}+\cdots+l_{k} s_{k}=n}} \frac{n !}{l_{1}^{s_{1}} \ldots l_{k}^{s_{k}} s_{1} ! \ldots s_{k} !} \times \\
& \times \int_{\mathbb{R}_{+}^{s_{1}+\cdots+s_{k}}} f^{(n)}(\underbrace{u_{1}, \ldots, u_{1}}_{l_{1}}, \ldots, \underbrace{u_{s_{1}}, \ldots, u_{s_{1}}}_{l_{1}}, \ldots, \underbrace{u_{s_{1}+\cdots+s_{k}}, \ldots, u_{s_{1}+\cdots+s_{k}}}_{l_{k}}) \times \\
& \times g^{(n)}(\underbrace{u_{1}, \ldots, u_{1}}_{l_{1}}, \ldots, \underbrace{u_{s_{1}}, \ldots, u_{s_{1}}}_{l_{1}}, \ldots, \underbrace{u_{s_{1}+\cdots+s_{k}}, \ldots, u_{s_{1}+\cdots+s_{k}}}_{l_{k}}) \times \\
& \times \eta^{l_{1}-1}\left(u_{1}\right) \ldots \eta^{l_{1}-1}\left(u_{s_{1}}\right) \eta^{l_{2}-1}\left(u_{s_{1}+1}\right) \ldots \eta^{l_{2}-1}\left(u_{s_{1}+s_{2}}\right) \ldots \\
& \ldots \eta^{l_{k}-1}\left(u_{s_{1}+\cdots+s_{k-1}+1}\right) \ldots \eta^{l_{k}-1}\left(u_{s_{1}+\cdots+s_{k}}\right) \\
& \times d u_{1} \ldots d u_{s_{1}+\cdots+s_{k}} .
\end{aligned}
$$

Let $|\cdot|_{\text {ext }}$ denote the norm generated by the scalar product $\langle\cdot, \cdot\rangle_{\text {ext }}$, i.e., $\left|f^{(n)}\right|_{\text {ext }}:=$ $:=\sqrt{\left\langle f^{(n)}, \overline{f^{(n)}}\right\rangle_{\text {ext }}}$. Denote by $\mathcal{H}_{\text {ext }}^{(n)}$ the closure of $\mathcal{D}_{\mathbb{C}}^{\widehat{\otimes} n}$ with respect to $|\cdot|_{\text {ext }}$. The space $\mathcal{H}_{\text {ext }}^{(n)}$ can be understood as an extension of $\mathcal{H}_{\mathbb{C}}^{\otimes} n$ in a generalized sense: let $F^{(n)} \in \mathcal{H}_{\mathbb{C}}^{\otimes}, \dot{F}^{(n)} \in F^{(n)}$ be a representative (a function) from the equivalence class $F^{(n)}$ with a "zero diagonale", i.e., $\dot{F}^{(n)}\left(u_{1}, \ldots, u_{n}\right)=0$ if there exist $i, j \in\{1, \ldots, n\}$ such that $i \neq j$ but $u_{i}=u_{j}$. The function $\dot{F}^{(n)}$ generates an equivalence class in $\mathcal{H}_{\mathrm{ext}}^{(n)}$ that can be identified with $F^{(n)}$ [73].

For $F^{(n)} \in \mathcal{H}_{\text {ext }}^{(n)}, n \in \mathbb{Z}_{+}$, we define $\left\langle P_{n}, F^{(n)}\right\rangle \in\left(L^{2}\right)$ as an $\left(L^{2}\right)$-limit

$$
\left\langle P_{n}, F^{(n)}\right\rangle:=\lim _{k \rightarrow \infty}\left\langle P_{n}, f_{k}^{(n)}\right\rangle,
$$


where $\mathcal{D}_{\mathbb{C}}^{\widehat{\otimes} n} \ni f_{k}^{(n)} \rightarrow F^{(n)}$ in $\mathcal{H}_{\text {ext }}^{(n)}$ as $k \rightarrow \infty$. The forthcoming statement easily follows from the construction of polynomials $\left\langle P_{n}, F^{(n)}\right\rangle$ (see also [108]).

Theorem 2.1. A function $F \in\left(L^{2}\right)$ if and only if there exists a sequence of kernels

$$
\left(F^{(n)} \in \mathcal{H}_{\text {ext }}^{(n)}\right)_{n=0}^{\infty}
$$

such that $F$ can be presented in the form

$$
F=\sum_{n=0}^{\infty}\left\langle P_{n}, F^{(n)}\right\rangle
$$

where the series converges in $\left(L^{2}\right)$, i.e., the $\left(L^{2}\right)$-norm of $F$

$$
\|F\|_{\left(L^{2}\right)}^{2}=\sum_{n=0}^{\infty} n !\left|F^{(n)}\right|_{\mathrm{ext}}^{2}<\infty .
$$

Moreover, the system $\left\{\left\langle P_{n}, F^{(n)}\right\rangle, F^{(n)} \in \mathcal{H}_{\mathrm{ext}}^{(n)}, n \in \mathbb{Z}_{+}\right\}$is an orthogonal basis in $\left(L^{2}\right)$ in the sense that for $F, G \in\left(L^{2}\right)$ of form (2.4)

$$
\langle\langle F, G\rangle\rangle=\sum_{n=0}^{\infty} n !\left\langle F^{(n)}, G^{(n)}\right\rangle_{\mathrm{ext}}
$$

2.3. A nonregular rigging of $\left(L^{2}\right)$. One can show [73] that the generalized Meixner measure satisfies conditions $1,2,3,4^{\prime}$ of the previous section, therefore one can consider (nonregular) chain (1.8) with the central space $\left(L^{2}\right), \chi=\exp , \alpha$ and $\gamma$ from (2.2); and all results of the "biorthogonal analysis" hold true in the "Meixner analysis". Note that since the generalized Meixner polynomials are orthogonal in $\left(L^{2}\right)$, natural orthogonal bases in the generalized function spaces $\left(\mathcal{H}_{-\tau}\right)_{-q, \exp , \gamma, \alpha}$ consist of generalized functions $\left\langle P_{m}, F^{(m)}\right\rangle:=\left(\mathcal{H}_{-\tau}\right)_{-q, \exp , \gamma, \alpha}-\lim _{k \rightarrow \infty}\left\langle P_{m}, f_{k}^{(m)}\right\rangle, m \in \mathbb{Z}_{+}$, where $F^{(m)} \in \mathcal{H}_{-\tau, \mathbb{C}}^{(m)}$ the negative space from the chain

$$
\mathcal{D}_{\mathbb{C}}^{\prime(m)} \supset \mathcal{H}_{-\tau, \mathbb{C}}^{(m)} \supset \mathcal{H}_{\mathrm{ext}}^{(m)} \supset \mathcal{H}_{\tau, \mathbb{C}}^{\widehat{\otimes} m} \supset \mathcal{D}_{\mathbb{C}}^{\widehat{\otimes} m}
$$

(see [73] for more details), $\mathcal{D}_{\mathbb{C}}^{\widehat{\otimes} m} \ni f_{k}^{(m)} \rightarrow F^{(m)}$ in $\mathcal{H}_{-\tau, \mathbb{C}}^{(m)}$ as $k \rightarrow \infty$. The interconnection between the generalized functions $\left\langle P_{m}, \cdot\right\rangle$ and $Q_{\mu, m}^{\exp , \gamma, \alpha}$ (see the previous section) is given by the formulas

$$
\left\langle P_{m}, F^{(m)}\right\rangle=Q_{\mu, m}^{\exp , \gamma, \alpha}\left(U_{m} F^{(m)}\right),
$$

where $\alpha$ and $\gamma$ from (2.2) and the operators $U_{m}: \mathcal{H}_{-\tau, \mathbb{C}}^{(m)} \rightarrow \mathcal{H}_{-\tau, \mathbb{C}}^{\widehat{\otimes} m}, m \in \mathbb{Z}_{+}$, are defined as follows:

$$
\forall f^{(m)} \in \mathcal{H}_{\tau, \mathbb{C}}^{\widehat{\otimes} m}\left\langle F^{(m)}, f^{(m)}\right\rangle_{\text {ext }}=\left\langle U_{m} F^{(m)}, f^{(m)}\right\rangle
$$


2.4. A parametrized regular rigging of $\left(L^{2}\right)$. Now we begin to observe some results of the "Meixner analysis" that can not be obtained as consequences of results of the "biorthogonal theory". First we introduce a so-called (regular) parametrized rigging of $\left(L^{2}\right)$. On the set $\mathcal{P}\left(\mathcal{D}^{\prime}\right)$ of all continuous polynomials on $\mathcal{D}^{\prime}$, presented as the set of functions (1.4) with $\chi=\exp , \alpha$ and $\gamma$ from (2.2) (i.e., now $P_{n}^{\chi, \gamma, \alpha}(x)=P_{n}(x)$ ), we introduce a family of Hilbert norms $\|\cdot\|_{q, \beta}, q \in \mathbb{Z}_{+}, \beta \in[0,1]$ (in what follows, we accept these conditions on default), by setting for $f$ of form (1.4)

$$
\|f\|_{q, \beta}^{2}:=\sum_{n=0}^{N_{f}}(n !)^{1+\beta} 2^{q n}\left|f^{(n)}\right|_{\mathrm{ext}}^{2} .
$$

By $\left(L^{2}\right)_{q}^{\beta}$ denote a Hilbert space that is the closure of $\mathcal{P}\left(\mathcal{D}^{\prime}\right)$ with respect to norm (2.6). Let also $\left(L^{2}\right)^{\beta}:=\operatorname{pr} \lim _{q \in \mathbb{Z}_{+}}\left(L^{2}\right)_{q}^{\beta}$. The spaces $\left(L^{2}\right)_{q}^{\beta},\left(L^{2}\right)^{\beta}$ are called the parametrized Kondratiev-type test function spaces. It is easy to see that $f \in\left(L^{2}\right)_{q}^{\beta}$ if and only if $f$ can be presented in the form

$$
f=\sum_{n=0}^{\infty}\left\langle P_{n}, f^{(n)}\right\rangle, \quad f^{(n)} \in \mathcal{H}_{\mathrm{ext}}^{(n)},
$$

with

$$
\|f\|_{\left(L^{2}\right)_{q}^{\beta}}^{2}=\sum_{n=0}^{\infty}(n !)^{1+\beta} 2^{q n}\left|f^{(n)}\right|_{\mathrm{ext}}^{2}<\infty .
$$

It is easy to show [73] that for arbitrary $q \in \mathbb{Z}_{+}$and $\beta \in[0,1]$ the space $\left(L^{2}\right)_{q}^{\beta}$ is densely and continuously embedded into $\left(L^{2}\right)$, therefore one can consider the chain

$$
\left(L^{2}\right)^{-\beta}=\operatorname{ind}_{q^{\prime} \in \mathbb{Z}_{+}} \lim _{-}\left(L^{2}\right)_{-q^{\prime}}^{-\beta} \supset\left(L^{2}\right)_{-q}^{-\beta} \supset\left(L^{2}\right) \supset\left(L^{2}\right)_{q}^{\beta} \supset\left(L^{2}\right)^{\beta},
$$

where $\left(L^{2}\right)_{-q}^{-\beta},\left(L^{2}\right)^{-\beta}$ are the spaces dual of $\left(L^{2}\right)_{q}^{\beta},\left(L^{2}\right)^{\beta}$ with respect to $\left(L^{2}\right)$ correspondingly. The spaces $\left(L^{2}\right)_{-q}^{-\beta},\left(L^{2}\right)^{-\beta}$ are called the parametrized Kondratievtype (regular) generalized function spaces. Note that for $\beta=q=0\left(L^{2}\right)_{0}^{0}=\left(L^{2}\right)_{-0}^{-0}=$ $=\left(L^{2}\right)$.

Since the generalized Meixner polynomials are orthogonal in $\left(L^{2}\right)$, these polynomials form orthogonal bases in $\left(L^{2}\right)_{-q}^{-\beta}$. More exactly, a function $F \in\left(L^{2}\right)_{-q}^{-\beta}$ if and only if there exists sequence (2.3) such that $F$ can be presented in form (2.4) with

$$
\|F\|_{-q,-\beta}^{2}:=\|F\|_{\left(L^{2}\right)_{-q}^{-\beta}}^{2}=\sum_{n=0}^{\infty}(n !)^{1-\beta} 2^{-q n}\left|F^{(n)}\right|_{\mathrm{ext}}^{2}<\infty .
$$

2.5. Elements of a Wick calculus. Now we consider elements of a Wick calculus on the parametrized generalized function spaces. For $F \in\left(L^{2}\right)^{-\beta}$ we define the $S$ transform $(S F)(\lambda), \lambda \in \mathcal{D}_{\mathbb{C}}$, as a formal series

$$
(S F)(\lambda):=\sum_{n=0}^{\infty}\left\langle F^{(n)}, \lambda^{\otimes n}\right\rangle_{\mathrm{ext}},
$$

where $F^{(n)} \in \mathcal{H}_{\text {ext }}^{(n)}, n \in \mathbb{Z}_{+}$, are the kernels from decomposition (2.4) for $F$. It is obvious that, in particular, $(S F)(0)=F^{(0)}, S 1 \equiv 1$. Further, for $F, G \in\left(L^{2}\right)^{-\beta}$ and 
a holomorphic at $F^{(0)}$ function $h: \mathbb{C} \rightarrow \mathbb{C}$ by analogy with the "biorthogonal case" we define a Wick product $F \diamond G$ and a Wick version $h^{\diamond}(F)$ by seting formally

$$
F \diamond G:=S^{-1}(S F \cdot S G), \quad h^{\diamond}(F):=S^{-1} h(S F) .
$$

A formal calculation shows that

$$
\begin{gathered}
F \diamond G=\sum_{m=0}^{\infty}\left\langle P_{m}, \sum_{k=0}^{m} F^{(k)} \diamond G^{(m-k)}\right\rangle, \\
F_{1} \diamond \ldots \diamond F_{n}=\sum_{m=0}^{\infty}\left\langle P_{m}, \sum_{\substack{k_{1}, \ldots, k_{n} \in \mathbb{Z}_{+}, k_{1}+\cdots+k_{n}=m}} F_{1}^{\left(k_{1}\right)} \diamond \cdots \diamond F_{n}^{\left(k_{n}\right)}\right\rangle, \\
h^{\diamond}(F)=h_{0}+\sum_{m=1}^{\infty}\left\langle P_{m}, \sum_{n=1}^{m} h_{n} \sum_{\substack{k_{1}, \ldots, k_{n} \in \mathbb{N}, k_{1}+\ldots+k_{n}=m}} F^{\left(k_{1}\right)} \diamond \cdots \diamond F^{\left(k_{n}\right)}\right\rangle
\end{gathered}
$$

(cf. (1.14)-(1.16)), where $F^{(k)}, G^{(k)}, F_{1}^{(k)}, \ldots, F_{n}^{(k)} \in \mathcal{H}_{\text {ext }}^{(k)}$ are the kernels from decompostions (2.4) for $F, G, F_{1}, \ldots, F_{n}$ correspondingly; $h_{n} \in \mathbb{C}, n \in \mathbb{Z}_{+}$, are the coefficients from the decomposition $h(u)=\sum_{n=0}^{\infty} h_{n}\left(u-F^{(0)}\right)^{n}$; for $F^{(n)} \in \mathcal{H}_{\text {ext }}^{(n)}$, $G^{(m)} \in \mathcal{H}_{\mathrm{ext}}^{(m)}$

$$
F^{(n)} \diamond G^{(m)}:=U_{n+m}^{-1}\left(\left(U_{n} F^{(n)}\right) \widehat{\otimes}\left(U_{m} G^{(m)}\right)\right) \in \mathcal{H}_{\mathrm{ext}}^{(n+m)},
$$

see (2.5). The fact that $F^{(n)} \diamond G^{(m)} \in \mathcal{H}_{\mathrm{ext}}^{(n+m)}$ is proved in [73]; moreover, it is shown therein that, roughly speaking, $F^{(n)} \diamond G^{(m)}$ is a symmetrization with respect to all variables of a function

$$
\begin{cases}F^{(n)}\left(u_{1}, \ldots, u_{n}\right) G^{(m)}\left(u_{n+1}, \ldots, u_{n+m}\right), & \text { if } \underset{\forall j \in\{n+1, \ldots, n\},}{\forall i \in+m\} u_{i} \neq u_{j}}, \\ 0, & \text { in other cases }\end{cases}
$$

and $\left|F^{(n)} \diamond G^{(m)}\right|_{\text {ext }} \leq\left|F^{(n)}\right|_{\text {ext }}\left|G^{(m)}\right|_{\text {ext }}$.

The forthcoming theorem from results of $[66,73]$ follows.

Theorem 2.2. The following statements are fulfilled:

Let $F, G \in\left(L^{2}\right)^{-\beta}$. Then $F \triangleright G \in\left(L^{2}\right)^{-\beta}$. Moreover, the Wick product is continuous in the topology of $\left(L^{2}\right)^{-\beta}$ : for arbitrary $F_{1}, \ldots, F_{n} \in\left(L^{2}\right)^{-\beta}$ there exist (depending on these elements $) q, q^{\prime} \in \mathbb{Z}_{+}\left(q>q^{\prime}+(1-\beta) \log _{2} n+1\right)$ such that

$$
\left\|F_{1} \diamond \ldots \diamond F_{n}\right\|_{-q,-\beta} \leq c(n-1)\left\|F_{1}\right\|_{-q^{\prime},-\beta} \ldots\left\|F_{n}\right\|_{-q^{\prime},-\beta},
$$

where $c(n):=\sqrt{\max _{m \in \mathbb{Z}_{+}}\left[2^{-m}(m+1)^{n}\right]}$.

For $F \in\left(L^{2}\right)^{-1}$ and a holomorphic at $(S F)(0)$ function $h: \mathbb{C} \rightarrow \mathbb{C} h^{\diamond}(F) \in$ $\in\left(L^{2}\right)^{-1}$.

Let $h: \mathbb{C} \rightarrow \mathbb{C}$ be a holomorphic at $u_{0} \in \mathbb{C}$ not-polynomial function with nonnegative coefficients $h_{n}$ from the decomposition $h(u)=\sum_{n=0}^{\infty} h_{n}\left(u-u_{0}\right)^{n}$. Then for each $\beta \in[0,1)$ there exists $F \in\left(L^{2}\right)^{-\beta}$ with $(S F)(0)=u_{0}$ such that $h^{\diamond}(F) \notin\left(L^{2}\right)^{-\beta}$. 
Let $F=\sum_{m=0}^{N}\left\langle P_{m}, F^{(m)}\right\rangle, F^{(m)} \in \mathcal{H}_{\mathrm{ext}}^{(m)} ; h: \mathbb{C} \rightarrow \mathbb{C}$ be a holomorphic at $F^{(0)}$ function such that the coefficients $h_{n}$ from the decomposition $h(u)=\sum_{n=0}^{\infty} h_{n}(u-$ $\left.-F^{(0)}\right)^{n}$ satisfy the estimates

$$
\left|h_{n}\right| \leq \frac{K^{n}}{n^{n N \frac{1-\beta}{2}}}
$$

with some $K>0$. Then $h^{\diamond}(F) \in\left(L^{2}\right)^{-\beta}$.

Note that the proof of this theorem is based on "coordinate" representations (2.8).

Finally, if $F, G \in\left(L^{2}\right)^{-\beta} \cap(\mathcal{D})_{\exp , \mu}^{\prime}$ and $\alpha, \gamma$ are defined in (2.2) then $F \diamond G=$ $=F \diamond_{\mu}^{\exp , \gamma, \alpha} G$, see [73].

2.6. An extended stochastic integral. By analogy with the Gaussian analysis, on the probability triplet $\left(\mathcal{D}^{\prime}, \mathcal{F}\left(\mathcal{D}^{\prime}\right), \mu\right)$ we define the Meixner random process $M$ by setting for each $u \in \mathbb{R}_{+} M_{u}:=\left\langle P_{1}, 1_{[0, u)}\right\rangle \in\left(L^{2}\right)$. Using results of [108] one can show that $M$ is a locally square integrable normal martingale (with respect to the generated by $M$ flow of $\sigma$-algebras) with orthogonal independent increments. Note that $M$ is not a Lévy process, generally speaking (not time-homogeneous). Let us construct an extended (Skorohod-type) stochastic integral with respect to $M$. Let $F \in\left(L^{2}\right)_{-q}^{-\beta} \otimes \mathcal{H}_{\mathbb{C}}$. It follows from above-posed results that $F$ can be presented in the form

$$
F(\cdot)=\sum_{m=0}^{\infty}\left\langle P_{m}, F^{(m)}\right\rangle, \quad F_{\cdot}^{(m)} \in \mathcal{H}_{\mathrm{ext}}^{(m)} \otimes \mathcal{H}_{\mathbb{C}}
$$

with

$$
\|F\|_{\left(L^{2}\right)_{-q}^{-\beta} \otimes \mathcal{H}_{\mathbb{C}}}^{2}=\sum_{m=0}^{\infty}(m !)^{1-\beta} 2^{-q m}\left|F^{(m)}\right|_{\mathcal{H}_{\mathrm{ext}}^{(m)} \otimes \mathcal{H}_{\mathbb{C}}}^{2}<\infty .
$$

If in addition $F$ is such that the kernels $F^{(m)}$ belong to $\mathcal{H}_{\mathbb{C}}^{\widehat{\otimes} m} \otimes \mathcal{H}_{\mathbb{C}} \subset \mathcal{H}_{\text {ext }}^{(m)} \otimes \mathcal{H}_{\mathbb{C}}$ (the embedding in the generalized sense described above) then one can show [73] that $F$ can be presented in the form

$$
F(\cdot)=\sum_{m=0}^{\infty} m ! \int_{0}^{\infty} \int_{0}^{u_{m}} \ldots \int_{0}^{u_{2}} F^{(m)}\left(u_{1}, \ldots, u_{m}\right) d M_{u_{1}} \ldots d M_{u_{m}}
$$

i.e., as a series of repeated Itô stochastic integrals with respect to the Meixner process. In this case for arbitrary $t_{1}, t_{2} \in[0,+\infty], t_{1}<t_{2}$, one can define the extended stochastic integral of $F$ with respect to $M$ on $\left[t_{1}, t_{2}\right)$ as

$$
\begin{gathered}
\int_{t_{1}}^{t_{2}} F(u) \widehat{d} M_{u}:= \\
:=\sum_{m=0}^{\infty}(m+1) ! \int_{0}^{\infty} \int_{0}^{u} \ldots \int_{0}^{u_{2}} \widehat{F}_{\left[t_{1}, t_{2}\right)}^{(m)}\left(u_{1}, \ldots, u_{m}, u\right) d M_{u_{1}} \ldots d M_{u_{m}} d M_{u}=
\end{gathered}
$$

ISSN 1027-3190. Укр. мат. журн., 2010, m. 62, № 9 


$$
=\sum_{m=0}^{\infty}\left\langle P_{m+1}, \widehat{F}_{\left[t_{1}, t_{2}\right)}^{(m)}\right\rangle \in\left(L^{2}\right)_{-q-1}^{-\beta},
$$

where $\widehat{F}_{\left[t_{1}, t_{2}\right)}^{(m)} \in \mathcal{H}_{\mathbb{C}}^{\widehat{\otimes} m+1} \subset \mathcal{H}_{\text {ext }}^{(m+1)}$ is the projection of $F_{\cdot}^{(m)} 1_{\left[t_{1}, t_{2}\right)}(\cdot)$ onto $\mathcal{H}_{\mathbb{C}}^{\widehat{\otimes} m+1}$. In a general case such a definition can not be accepted because it is impossible to project elements of $\mathcal{H}_{\text {ext }}^{(m)} \otimes \mathcal{H}_{\mathbb{C}}$ onto $\mathcal{H}_{\text {ext }}^{(m+1)}$, generally speaking. Nevertheless, the following generalization is possible.

Lemma 2.1 ([73]). For given $F^{(m)} \in \mathcal{H}_{\text {ext }}^{(m)} \otimes \mathcal{H}_{\mathbb{C}}, m \in \mathbb{Z}_{+}$, and $t_{1}, t_{2} \in$ $[0,+\infty], t_{1}<t_{2}$, we construct the element $\widehat{F}_{\left[t_{1}, t_{2}\right)}^{(m)} \in \mathcal{H}_{\mathrm{ext}}^{(m+1)}$ by the following way. Let $\dot{F}^{(m)} \in F^{(m)}$ be some representative (a function) from the equivalence class $F_{.}^{(m)}$. We set

$\widetilde{\dot{F}}_{\left[t_{1}, t_{2}\right)}^{(m)}\left(u_{1}, \ldots, u_{m}, u\right):= \begin{cases}\dot{F}_{u}^{(m)}\left(u_{1}, \ldots, u_{m}\right) 1_{\left[t_{1}, t_{2}\right)}(u), & \text { if } u \neq u_{1}, \ldots, u \neq u_{m} \\ 0, & \text { in other cases }\end{cases}$ $\widehat{\dot{F}}_{\left[t_{1}, t_{2}\right)}^{(m)}:=\operatorname{Pr} \widetilde{\dot{F}}_{\left[t_{1}, t_{2}\right)}^{(m)}$, where $\operatorname{Pr}$ is the symmetrization operator. Let $\widehat{F}_{\left[t_{1}, t_{2}\right)}^{(m)} \in \mathcal{H}_{\mathrm{ext}}^{(m+1)}$ be the equivalence class in $\mathcal{H}_{\mathrm{ext}}^{(m+1)}$ that is generated by $\widehat{\dot{\vec{F}}}_{\left[t_{1}, t_{2}\right)}^{(m)}$. This class is welldefined, does not depend on the representative $\dot{F}^{(m)}$, and

$$
\left|\widehat{F}_{\left[t_{1}, t_{2}\right)}^{(m)}\right|_{\text {ext }} \leq\left|F^{(m)} 1_{\left[t_{1}, t_{2}\right)}(\cdot)\right|_{\mathcal{H}_{\mathrm{ext}}^{(m)} \otimes \mathcal{H}_{\mathbb{C}}} \leq\left|F^{(m)}\right|_{\mathcal{H}_{\mathrm{ext}}^{(m)} \otimes \mathcal{H}_{\mathbb{C}}} .
$$

Now for $F \in\left(L^{2}\right)_{-q}^{-\beta} \otimes \mathcal{H}_{\mathbb{C}}$ of form (2.10) we can define the extended stochastic integral on $\left[t_{1}, t_{2}\right)$ with respect to $M$ by setting [73]

$$
\int_{t_{1}}^{t_{2}} F(u) \widehat{d} M_{u}:=\sum_{m=0}^{\infty}\left\langle P_{m+1}, \widehat{F}_{\left[t_{1}, t_{2}\right)}^{(m)}\right\rangle \in\left(L^{2}\right)_{-q-1}^{-\beta} .
$$

If we consider the extended stochastic integral as an operator acting from $\left(L^{2}\right)_{-q}^{-\beta} \otimes \mathcal{H}_{\mathbb{C}}$ to $\left(L^{2}\right)_{-q}^{-\beta}$ (for example, if $\beta=q=0$ then it will be an operator acting from $\left(L^{2}\right) \otimes \mathcal{H}_{\mathbb{C}}$ to $\left(L^{2}\right)$ ) then for $\beta<1$ this operator will be unbounded with the domain

$$
\left\{F \in\left(L^{2}\right)_{-q}^{-\beta} \otimes \mathcal{H}_{\mathbb{C}}: \sum_{m=0}^{\infty}((m+1) !)^{1-\beta} 2^{-q(m+1)}\left|\widehat{F}_{\left[t_{1}, t_{2}\right)}^{(m)}\right|_{\text {ext }}^{2}<\infty\right\} .
$$

Theorem 2.3 ([73]). Let $F \in\left(L^{2}\right) \otimes \mathcal{H}_{\mathbb{C}}$ be integrable on $\mathbb{R}_{+}$by Itô with respect to $M$ (i.e., be adapted with respect to the generated by $M$ flow of $\sigma$-algebras). Then for all $t_{1}, t_{2} \in[0,+\infty], t_{1}<t_{2}, F$ is integrable on $\left[t_{1}, t_{2}\right)$ by Ito and in the extended sense, and $\int_{t_{1}}^{t_{2}} F(u) \widehat{d} M_{u}=\int_{t_{1}}^{t_{2}} F(u) d M_{u}$ (the last integral is the Itô one).

Let us consider main properties of the extended stochastic integral. First we note that if $t_{1}, t_{2}, t_{3} \in[0,+\infty]$ and $t_{1}<t_{2}<t_{3}$ then $\int_{t_{1}}^{t_{2}} \circ(u) \widehat{d} M_{u}+\int_{t_{2}}^{t_{3}} \circ(u) \widehat{d} M_{u}=$ $=\int_{t_{1}}^{t_{3}} \circ(u) \widehat{d} M_{u}$; but applying this formula one has to keep in mind that for $\beta<1$ 
the domain of $\int_{a}^{b} \circ(u) \widehat{d} M_{u}:\left(L^{2}\right)_{-q}^{-\beta} \otimes \mathcal{H}_{\mathbb{C}} \rightarrow\left(L^{2}\right)_{-q}^{-\beta}$ depends on the integration interval $[a, b)$. Further, taking into consideration that each $g^{(n)} \in \mathcal{H}_{\mathrm{ext}}^{(n)}, n \in \mathbb{N}$, can be considered as $g^{(n)}(\cdot) \in \mathcal{H}_{\text {ext }}^{(n-1)} \otimes \mathcal{H}_{\mathbb{C}}$ with $\left|g^{(n)}(\cdot)\right|_{\mathcal{H}_{\text {ext }}^{(n-1)} \otimes \mathcal{H}_{\mathbb{C}}} \leq\left|g^{(n)}\right|_{\text {ext }}[$ [73], we define the (Hida-type) stochastic derivative $1_{\left[t_{1}, t_{2}\right)}(\cdot) \partial .:\left(L^{2}\right)_{q+1}^{\beta} \rightarrow\left(L^{2}\right)_{q}^{\beta} \otimes \mathcal{H}_{\mathbb{C}}$, $t_{1}, t_{2} \in[0,+\infty], t_{1}<t_{2}$, by setting for $g \in\left(L^{2}\right)_{q+1}^{\beta}$ of form (2.7)

$$
1_{\left[t_{1}, t_{2}\right)}(\cdot) \partial . g:=\sum_{n=0}^{\infty}(n+1)\left\langle P_{n}, g^{(n+1)}(\cdot) 1_{\left[t_{1}, t_{2}\right)}(\cdot)\right\rangle .
$$

Of course, one can understand $1_{\left[t_{1}, t_{2}\right)}(\cdot) \partial$. as a linear unbounded operator acting from $\left(L^{2}\right)_{q}^{\beta}$ to $\left(L^{2}\right)_{q}^{\beta} \otimes \mathcal{H}_{\mathbb{C}}$ with the domain

$$
\begin{gathered}
\left\{g=\sum_{n=0}^{\infty}\left\langle P_{n}, g^{(n)}\right\rangle \in\left(L^{2}\right)_{q}^{\beta}:\right. \\
\left.\sum_{n=0}^{\infty}(n !)^{1+\beta} 2^{q n}(n+1)^{2}\left|g^{(n+1)}(\cdot) 1_{\left[t_{1}, t_{2}\right)}(\cdot)\right|_{\mathcal{H}_{\text {ext }}^{(n)} \otimes \mathcal{H}_{\mathbb{C}}}^{2}<\infty\right\} .
\end{gathered}
$$

Theorem 2.4 ([73]). For all $t_{1}, t_{2} \in[0,+\infty], t_{1}<t_{2}$, the operators $\int_{t_{1}}^{t_{2}} \circ(u) \widehat{d} M_{u}$ : $\left(L^{2}\right)_{-q}^{-\beta} \otimes \mathcal{H}_{\mathbb{C}} \rightarrow\left(L^{2}\right)_{-q}^{-\beta}$ and $1_{\left[t_{1}, t_{2}\right)}(\cdot) \partial .:\left(L^{2}\right)_{q}^{\beta} \rightarrow\left(L^{2}\right)_{q}^{\beta} \otimes \mathcal{H}_{\mathbb{C}}$ are adjoint one to another:

$$
\int_{t_{1}}^{t_{2}} \circ(u) \widehat{d} M_{u}=\left(1_{\left[t_{1}, t_{2}\right)}(\cdot) \partial .\right)^{*} ; \quad 1_{\left[t_{1}, t_{2}\right)}(\cdot) \partial .=\left(\int_{t_{1}}^{t_{2}} \circ \widehat{d} M\right)^{*} .
$$

In particular, these operators are closed.

Of course, the statement of this theorem holds true if we consider $\int_{t_{1}}^{t_{2}} \circ(u) \widehat{d} M_{u}:\left(L^{2}\right)_{-q}^{-\beta} \otimes$ $\mathcal{H}_{\mathbb{C}} \rightarrow\left(L^{2}\right)_{-q-1}^{-\beta}$ and $1_{\left[t_{1}, t_{2}\right)}(\cdot) \partial .:\left(L^{2}\right)_{q+1}^{\beta} \rightarrow\left(L^{2}\right)_{q}^{\beta} \otimes \mathcal{H}_{\mathbb{C}}$

By analogy one can consider the extended stochastic integral as a linear continuous operator acting from $\left(L^{2}\right)^{-\beta} \otimes \mathcal{H}_{\mathbb{C}}$ to $\left(L^{2}\right)^{-\beta}$.

Let us consider now the interconnection between the Wick calculus and the extended stochastic integration. Let $M_{u}^{\prime}=\left\langle P_{1}, \delta_{u}\right\rangle \in\left(\mathcal{H}_{-\tau}\right)_{-q, \exp , \gamma, \alpha, \mu}$ (see (1.8); now $\alpha$ and $\gamma$ from (2.2)) be the Meixner white noise [73, 108].

Theorem 2.5 ([73]). For all $t_{1}, t_{2} \in[0,+\infty], t_{1}<t_{2}$, and $F \in\left(L^{2}\right)^{-\beta} \otimes \mathcal{H}_{\mathbb{C}}$ the formally defined Pettis integral $\int_{t_{1}}^{t_{2}} F(u) \diamond M_{u}^{\prime} d u$ can be considered as a linear continuous functional on $\left(L^{2}\right)^{\beta}$ that coincides with $\int_{t_{1}}^{t_{2}} F(u) \widehat{d} M_{u}$, i.e.,

$$
\int_{t_{1}}^{t_{2}} F(u) \diamond M_{u}^{\prime} d u=\int_{t_{1}}^{t_{2}} F(u) \widehat{d} M_{u} \in\left(L^{2}\right)^{-\beta}
$$


Let us consider an example of solving of an equation with Wick-type nonlinearity. Let

$$
X_{t}=X_{0}+\int_{0}^{t} X_{u} \diamond F d u+\int_{0}^{t} X_{u} \diamond G \widehat{d} M_{u},
$$

where $X_{0}, F, G \in\left(L^{2}\right)^{-\beta}$. Applying the $S$-transform, solving the obtained nonstochastic equation and applying the inverse $S$-transform we obtain the solution

$$
X_{t}=X_{0} \diamond \exp ^{\diamond}\left\{F t+G \diamond M_{t}\right\} \in\left(L^{2}\right)^{-1} .
$$

In order to have $X_{t} \in\left(L^{2}\right)^{-\beta}, \beta<1$, we need additional restrictions. For example, if $F$ and $G$ are polynomials and $N:=\max \{\operatorname{deg} F, \operatorname{deg} G+1\}$, where $\operatorname{deg}$ denotes the degree of a polynomial, then $X_{t} \in\left(L^{2}\right)^{-\beta}$ if $N \leq \frac{2}{1-\beta}$ [66].

Finally we note that if $F \in\left(L^{2}\right)^{-\beta} \otimes \mathcal{H}_{\mathbb{C}} \cap(\mathcal{D})_{\exp , \mu}^{\prime} \otimes \mathcal{H}_{\mathbb{C}}$ and $\alpha$ is defined in (2.2) then for all $t_{1}, t_{2} \in[0,+\infty], t_{1}<t_{2}$,

$$
\int_{t_{1}}^{t_{2}} F(u) \widehat{d} M_{u}=\mathbf{I}_{\left[t_{1}, t_{2}\right)}^{\exp , \alpha, \mu}(F),
$$

this result is proved in [73].

2.7. Operators of stochastic differentiation. In contrast to the general "biorthogonal" case, now the operator $1_{\left[t_{1}, t_{2}\right)}(\cdot) \partial ., t_{1}, t_{2} \in[0,+\infty], t_{1}<t_{2}$, can be naturally continued to the generalized function spaces. More exactly, for $F \in\left(L^{2}\right)_{-q}^{-\beta}$ of form (2.4) we define $1_{\left[t_{1}, t_{2}\right)}(\cdot) \partial . F \in\left(L^{2}\right)_{-q-1}^{-\beta} \otimes \mathcal{H}_{\mathbb{C}}$ by setting (cf. (2.11))

$$
1_{\left[t_{1}, t_{2}\right)}(\cdot) \partial . F:=\sum_{m=0}^{\infty}(m+1)\left\langle P_{m}, F^{(m+1)}(\cdot) 1_{\left[t_{1}, t_{2}\right)}(\cdot)\right\rangle .
$$

This operator can be naturally continued to a linear continuous operator acting from $\left(L^{2}\right)^{-\beta}$ to $\left(L^{2}\right)^{-\beta} \otimes \mathcal{H}_{\mathbb{C}}$; also one can consider $1_{\left[t_{1}, t_{2}\right)}(\cdot) \partial$. as a linear unbounded operator acting from $\left(L^{2}\right)_{-q}^{-\beta}$ to $\left(L^{2}\right)_{-q}^{-\beta} \otimes \mathcal{H}_{\mathbb{C}}$ with the domain consists of $F \in\left(L^{2}\right)_{-q}^{-\beta}$ such that $\left\|1_{\left[t_{1}, t_{2}\right)}(\cdot) \partial . F\right\|_{\left(L^{2}\right)_{-q}^{-\beta} \otimes \mathcal{H}_{\mathbb{C}}}<\infty$, in this case $1_{\left[t_{1}, t_{2}\right)}(\cdot) \partial$. is closed [71].

Properties of $1_{\left[t_{1}, t_{2}\right)}(\cdot) \partial$. on the generalized function spaces are similar to properties of $1_{\left[t_{1}, t_{2}\right)}(\cdot) \partial$. on the test function spaces. In particular, $1_{\left[t_{1}, t_{2}\right)}(\cdot) \partial$. is the adjoint operator to the restriction of the extended stochastic integral on the corresponding test function space. The interested reader can find a more detailed information in [71].

Now let us consider operators of stochastic differentiation on the generalized function spaces. For $F^{(m)} \in \mathcal{H}_{\text {ext }}^{(m)}$ and $f^{(n)} \in \mathcal{H}_{\text {ext }}^{(n)}, m>n$, we define a "pairing" $\left\langle F^{(m)}, f^{(n)}\right\rangle_{\mathrm{ext}} \in \mathcal{H}_{\mathrm{ext}}^{(m-n)}$ by setting for each $g^{(m-n)} \in \mathcal{H}_{\mathrm{ext}}^{(m-n)}$

$$
\left\langle\left\langle F^{(m)}, f^{(n)}\right\rangle_{\mathrm{ext}}, g^{(m-n)}\right\rangle_{\mathrm{ext}}=\left\langle F^{(m)}, f^{(n)} \diamond g^{(m-n)}\right\rangle_{\mathrm{ext}}
$$

(see (2.9)). One can show [71] that for arbitrary $F^{(m)} \in \mathcal{H}_{\text {ext }}^{(m)}$ and $f^{(1)} \in \mathcal{H}_{\text {ext }}^{(1)}=\mathcal{H}_{\mathbb{C}}$

$$
\left\langle F^{(m)}, f^{(1)}\right\rangle_{\mathrm{ext}}=\int_{\mathbb{R}_{+}} F^{(m)}(u) f^{(1)}(u) d u,
$$

where the integral in the right-hand side is a Pettis one. 
For arbitrary $f^{(n)} \in \mathcal{H}_{\mathrm{ext}}^{(n)}, n \in \mathbb{Z}_{+}$, we define a linear operator $\left(\mathbb{D}^{n} \circ\right)\left(f^{(n)}\right)$ : $\left(L^{2}\right)_{-q}^{-\beta} \rightarrow\left(L^{2}\right)_{-q-1}^{-\beta}$ by setting for $F \in\left(L^{2}\right)_{-q}^{-\beta}$

$$
\left(\mathbb{D}^{n} F\right)\left(f^{(n)}\right):=\sum_{m=0}^{\infty} \frac{(m+n) !}{m !}\left\langle P_{m},\left\langle F^{(m+n)}, f^{(n)}\right\rangle_{e x t}\right\rangle,
$$

where $F^{(m)} \in \mathcal{H}_{\mathrm{ext}}^{(m)}, m \in \mathbb{Z}_{+}$, are the kernels from decomposition (2.4) for $F$. It follows from results of [71] that $\left(\mathbb{D}^{n} \circ\right)\left(f^{(n)}\right)$ is a continuous operator that can be continued to a linear continuous operator in $\left(L^{2}\right)^{-\beta}$, and the following statement is fulfilled.

Theorem 2.6. The operator $\mathbb{D}^{n}$ has the following properties:

for $g_{1}^{(1)}, \ldots, g_{n}^{(1)} \in \mathcal{H}_{\mathrm{ext}}^{(1)}=\mathcal{H}_{\mathbb{C}}$

$$
\underbrace{\left(\mathbb { D } ^ { 1 } \left(\ldots \left(\mathbb { D } ^ { 1 } \left(\left(\mathbb{D}^{1} \circ\right)\right.\right.\right.\right.}_{n}\left(g_{1}^{(1)}\right)))\left(g_{2}^{(1)}\right) \ldots))\left(g_{n}^{(1)}\right)=\left(\mathbb{D}^{n} F\right)\left(g_{1}^{(1)} \diamond \cdots \diamond g_{n}^{(1)}\right) ;
$$

for each $F \in\left(L^{2}\right)_{-q}^{-\beta}$ the kernels $F^{(n)} \in \mathcal{H}_{\mathrm{ext}}^{(n)}$ from decomposition (2.4) can be presented in the form

$$
F^{(n)}=\frac{1}{n !} \mathbf{E}\left(\mathbb{D}^{n} F\right)
$$

i.e., for each $f^{(n)} \in \mathcal{H}_{\mathrm{ext}}^{(n)}\left\langle F^{(n)}, f^{(n)}\right\rangle_{\mathrm{ext}}=\frac{1}{n !} \mathbf{E}\left[\left(\mathbb{D}^{n} F\right)\left(f^{(n)}\right)\right]$, here $\mathbf{E}$ denotes the expectation;

for all $F \in\left(L^{2}\right)_{-q}^{-\beta}$ and $f^{(1)} \in \mathcal{H}_{\mathrm{ext}}^{(1)}=\mathcal{H}_{\mathbb{C}}$

$$
\int_{\mathbb{R}_{+}} \partial_{u} F \cdot f^{(1)}(u) d u=\left(\mathbb{D}^{1} F\right)\left(f^{(1)}\right),
$$

here the integral in the left-hand side is a Pettis one;

the adjoint to $\mathbb{D}^{n}$ operator has a form

$$
\left(\mathbb{D}^{n} g\right)\left(f^{(n)}\right)^{*}=\sum_{m=0}^{\infty}\left\langle P_{m+n}, f^{(n)} \diamond g^{(m)}\right\rangle=g \diamond\left\langle P_{n}, f^{(n)}\right\rangle \in\left(L^{2}\right)_{q}^{\beta},
$$

where $g \in\left(L^{2}\right)_{q+1}^{\beta}, f^{(n)} \in \mathcal{H}_{\mathrm{ext}}^{(n)}$, and $g^{(m)} \in \mathcal{H}_{\mathrm{ext}}^{(m)}\left(m \in \mathbb{Z}_{+}\right)$are the kernels from decomposition (2.7) for $g$;

for all $F \in\left(L^{2}\right)_{-q}^{-\beta}, g \in\left(L^{2}\right)_{q+1}^{\beta}$ and $f^{(1)} \in \mathcal{H}_{\mathrm{ext}}^{(1)}=\mathcal{H}_{\mathbb{C}}$

$$
\begin{gathered}
\left\langle\left\langle F, \int_{\mathbb{R}_{+}} g \cdot f^{(1)}(u) \widehat{d} M_{u}\right\rangle\right\rangle=\left\langle\left\langle F, g \diamond\left\langle P_{1}, f^{(1)}\right\rangle\right\rangle\right\rangle= \\
=\left\langle\left\langle F,\left(\mathbb{D}^{1} g\right)\left(f^{(1)}\right)^{*}\right\rangle\right\rangle=\left\langle\left\langle\left(\mathbb{D}^{1} F\right)\left(f^{(1)}\right), g\right\rangle\right\rangle ;
\end{gathered}
$$

the operator $\mathbb{D}^{1}$ is a differentiation with respect to the Wick product, i.e., for all $F, G \in$ $\left(L^{2}\right)^{-\beta}$ and $g \in \mathcal{H}_{\mathrm{ext}}^{(1)}=\mathcal{H}_{\mathbb{C}}$

$$
\left(\mathbb{D}^{1}(F \diamond G)\right)(g)=\left(\mathbb{D}^{1} F\right)(g) \diamond G+F \diamond\left(\mathbb{D}^{1} G\right)(g) \in\left(L^{2}\right)^{-\beta} ;
$$


for any $n \in \mathbb{Z}_{+}, F \in\left(L^{2}\right)^{-\beta}, g \in \mathcal{H}_{\text {ext }}^{(1)}=\mathcal{H}_{\mathbb{C}}$, and a holomorphic at $(S F)(0)$ function $h: \mathbb{C} \rightarrow \mathbb{C}$

$$
\begin{aligned}
& \left(\mathbb{D}^{1} F^{\diamond n}\right)(g)=n F^{\diamond n-1} \diamond\left(\mathbb{D}^{1} F\right)(g) \in\left(L^{2}\right)^{-\beta}, \\
& \left(\mathbb{D}^{1} h^{\diamond}(F)\right)(g)=h^{\prime \diamond}(F) \diamond\left(\mathbb{D}^{1} F\right)(g) \in\left(L^{2}\right)^{-1},
\end{aligned}
$$

where $h^{\prime}$ is the usual derivative of $h$;

$$
\text { for all } t_{1}, t_{2} \in[0,+\infty], t_{1}<t_{2}, g \in \mathcal{H}_{\mathrm{ext}}^{(1)}=\mathcal{H}_{\mathbb{C}}, \text { and } F \in\left(L^{2}\right)^{-\beta} \otimes \mathcal{H}_{\mathbb{C}}
$$

$$
\left(\mathbb{D}^{1} \int_{t_{1}}^{t_{2}} F(u) \widehat{d} M_{u}\right)(g)=\int_{t_{1}}^{t_{2}}\left(\mathbb{D}^{1} F(u)\right)(g) \widehat{d} M_{u}+\int_{t_{1}}^{t_{2}} F(u) g(u) d u \in\left(L^{2}\right)^{-\beta},
$$

where the last integral in the right-hand side is a Pettis one.

An information about possible applications of the operators $\mathbb{D}^{n}$ and the corresponding examples can be found in, e.g., $[11,71]$.

One can consider the operator $\left(\mathbb{D}^{n} \circ\right)\left(f^{(n)}\right), f^{(n)} \in \mathcal{H}_{\text {ext }}^{(n)}$, as a linear unbounded one acting in $\left(L^{2}\right)_{-q}^{-\beta}$. This operator is closed and the analog of the previous theorem holds true (see details in [71]).

Finally we note that the operators $\widetilde{\mathbb{D}}_{\exp , \gamma, \alpha, \mu}^{n}\left(\alpha\right.$ and $\gamma$ from (2.2)) and $\mathbb{D}^{n}, n \in \mathbb{N}$, do not cioncide on the set $\left(L^{2}\right)^{-\beta} \cap(\mathcal{D})_{\exp , \mu}^{\prime}$, generally speaking (if $\eta \neq 0$, see (2.1)); but, as we saw, their properties are quite similar.

1. Accardi $L$. Meixner classes and the square of white noise // Contemp. Math. -2003 . - 137. - P. $1-13$.

2. Accardi L., Boukas A. The semi-martingale property of the square of white noise integrators // Lect. Notes Pure and Appl. Math. - 2002. - 227. - P. 1-19.

3. Accardi L., Bozéjko M. Interacting Fock space and Gaussianization of probability measures // Infinite Dimens. Anal. Quantum Probab. Relat. Top. - 1998. - 1, № 4. - P. $663-670$.

4. Accardi L., Fagnola F., Quaegebeur J. A representation free quantum stochastic calculus // J. Funct. Anal. - 1992. - 104, № 1. - P. 149-197.

5. Accardi L., Franz U., Skeide M. Renormalized squares of white noise and other non-Gaussian noises as Levy processes on real Lie algebras // Communs Math. Phys. - 2002. - 228. - P. 123-150.

6. Albeverio S., Berezansky Yu. M., Tesko V. A. A generalization of an extended stochastic integral // Ukr. Math. J. - 2007. - 59, № 5. - P. $645-677$.

7. Albeverio S., Daletsky Yu. L., Kondratiev Yu. G., Streit L. Non-Gaussian infnite-dimensional analysis // J. Funct. Anal. - 1996. - 138. - P. $311-350$.

8. Albeverio S., Kondratiev Yu. G., Streit $L$. How to generalize white noise analysis to non-Gaussian spaces // Proc. Simp. "Dynamics of Complex and Irregular Systems". - Singapore etc.: World Sci., 1993. P. $48-60$.

9. Anderson Ch. Some properties of Appell-like polynomials // J. Math. Anal. and Appl. - 1967. - 19. P. $475-491$.

10. Baklan V. V. On one generalization of a stochastic integral // Dokl. AN USSR. Ser. A. - 1976. S. $291-294$ (in Russian).

11. Benth F. E. The Gross derivative of generalized random variables // Infinite Dimens. Anal. Quantum Probab. Relat. Top. - 1999. - 2, № 3. - P. $381-396$.

12. Berezansky $Y$ и. M. Infinite-dimensional analysis related to generalized translation operator // Ukr. Math. J. - 1997. - 49, № 3. - P. $403-450$.

13. Berezansky $Y u$. M. Pascal measure on generalized functions and the corresponding generalized Meixner polynomials // Meth. Funct. Anal. and Top. - 2002. - 8, № 1. - P. 1-13.

14. Berezansky Yu. M. Self-adjoint operators in a space of functions depending on infinitely-many variables (in Russian). - K. Naukova Dumka, 1978. - 360 p.

15. Berezansky Yu. M. Spectral approach to white noise analysis // Proc. Simp. "Dynamics of Complex and Irregular Systems". - Singapore etc.: World Sci., 1993. - P. 131-140. 
16. Berezansky Yu. M., Kondratiev Yu. G. Biorthogonal systems in hypergroups: an extension of nonGaussian analysis // Meth. Funct. Anal. and Top. - 1996. - 2, № 2 - P. 1 - 50.

17. Berezansky Yu. M., Kondratiev Yu. G. Spectral methods in infinite-dimensional analysis (in Russian). K., Naukova Dumka, 1988. - 680 p.

18. Berezansky Yu. M., Livinsky V. D., Lytvynov E. W. Generalization of Gaussian white noise analysis // Meth. Funct. Anal. and Top. - 1995. - 1, № 1. - P. $28-55$.

19. Berezansky Yu. M., Lytvynov E. W., Mierzejewski D. A. The Jacobi field of a Lévy process // Ukr. Math. J. - 2003. - 55, № 5. - P. $853-858$.

20. Berezansky Yu. M., Merzejewski D. A. The structure of extended symmetric Fock space // Meth. Funct. Anal. and Top. - 2000. - 6, № 4. - P. 1-13.

21. Berezansky Yu. M., Samoilenko Yu. S. Nuclear spaces of fuctions of infinitely many variables // Ukr. Math. J. - 1973. - 25, № 6. - P. 599-609.

22. Berezansky Yu. M., Tesko V.A. An approach to a generalization of white noise analysis // Operator theory: Adv. and Appl. - 2009. - 190. - P. 123-139.

23. Berezansky Yu. M., Tesko V. A. Orthogonal approach to the construction of the theory of generalized functions of infinitely many variables and the Poisson analysis of White Noise // Ukr. Math. J. - 2004. - 56, № 12. - P. 1885 - 1914.

24. Berezansky Yu. M., Tesko V. A. Spaces of test and generalized functions related to generalized translation operators // Ibid. - 2003. - 55, № 12. - P. 1907-1979.

25. Berezansky Yu. M., Us G. F., Sheftel Z. G. Functional analysis. - Basel etc.: Birkh'auser, 1996. - 312 p.

26. Boas R. P., Buck R. C. Polynomial expansions of analytic functions. - Berlin: Springer, 1964. $\mathrm{VIII}+77 \mathrm{p}$.

27. Chung D. M., Chung T. S., Ji U. C. A characterization theorem for operators on white noise functionals // J. Math. Soc. Jap. - 1999. - 51, № 2. - P. 437-447.

28. Chung D. M., Ji U. C., Obata N. Higher powers of quantum white noises in terms of integral kernel operators // Infinite Dimens. Anal. Quantum Probab. Relat. Top. - 1998. - 1, № 4. - P. 533 - 559.

29. Chung D. M., Ji U. C., Obata N. Quantum stochastic analysis via white noise operators in weighted Fock space // Rev. Math. Phys. - 2002. - 14, № 3. - P. 241-272.

30. Daletsky Yu. L. A biorthogonal analog of the Hermite polynomials and the inversion of the Fourier transform with respect to a non-Gaussian measure // Funct. Anal. and Appl. - 1991. - 25, № 2. P. $138-140$.

31. Yu. L. Daletsky, Paramonova S. N. A certain formula of the theory of Gaussian measures, and the estimation of stochastic integrals // Teorija Verojatnostei i ee Pril. - 1974. - 19, № 4. - S. $845-849$ (in Russian).

32. Nunno G. Di, Meyer-Brandis T., Oksendal B., Proske F. Malliavin calculus and anticipative Itô formulae for Lévy processes // Infinite Dimens. Anal. Quantum Probab. Relat. Top. - 2005. - 8, № 2. - P. 235 258.

33. Nunno G. Di, Oksendal B., Proske F. Malliavin calculus for Lévi processes with applications to finance // Universitext. - Berlin: Springer, 2009.

34. Nunno G. Di, Oksendal B., Proske F. White noise analysis for Lévy processes // J. Funct. Anal. - 2004. - 206. - P. $109-148$.

35. Dineen $S$. Complex analysis in locally convex spaces // Math. Stud. - North-Holland, Amsterdam, 1981. - 57. - XIII+492 p.

36. Dorogovtsev A. A. An extended stochastic integral for smooth functionals of white noise // Ukr. Math. J. - 1989. - 41, № 11. - P. $1252-1258$.

37. Dorogovtsev A. A. A property of trajectories of extended stochastic integrals // Siberian Math. J. 1993. - 34, № 5. - P. 825 - 828 .

38. Dorogovtsev A. A. A stochastic integral with respect to the Arratia flow // Dokl. Akad. Nauk. -2006 - 410, № 2. - S. 156-157 (in Russian).

39. Dorogovtsev A. A. Boundary value problems for stochastic equations with anticipation // Dokl. Akad. Nauk. - 2008. - 418, № 4. - S. 443 - 446 (in Russian)

40. Dorogovtsev A. A. Fourier transform of Wiener functionals, and various methods of stochastic integration // Theor. Probab. Appl. - 1989. - 34, № 4. - P. $705-709$.

41. Dorogovtsev A. A. Elements of stochastic differential calculus // Math. Today '88. - K.: Vyshcha Shkola, 1988. - S. $105-131$ (in Russian). 
42. Dorogovtsev A. A. Equations with random Gaussian operators, and a stochastic calculus, Selected problems in the current theory of random processes (in Russian). - Kiev: Inst. Mat. Akad. Nauk Ukrain. SSR, 1988. - P. 52-60.

43. Dorogovtsev A. A. On the family of Ito formulas for the logarithmic processes. Asymptotic analysis of random evolutions (in Ukrainian). - Kiev: Inst. Mat. Akad. Nauk Ukrainy, 1994. - P. 100-112.

44. Dorogovtsev A. A. On the localization of an extended stochastic integral // Sb. Mat. - 2006. - 197, № 9-10. - S. $1273-1295$.

45. Dorogovtsev A. A. One version of the Clark representation theorem for Arratia flows // Theory Stochast. Process. - 2005. - 11, № 3-4. - P. 63-70.

46. Dorogovtsev A. A. Stochastic analysis and random mappings in a Hilbert space (in Russian). - K.: Naukova Dumka, 1992. - 120 p.

47. Dorogovtsev A. A. Stochastic equations with an anticipation. - Kyiv: Inst. Math. Nat. Acad. Sci. Ukraine, 1996. - $152 \mathrm{p}$.

48. Dorogovtsev A. A. Stochastic integrals with respect to compatible random measures // Ukr. Math. J. 2000. - 52, № 8. - P. 1215 - 1229 .

49. Dorogovtsev A. A. Stochastic integrals with respect to Gaussian random measures // Theor. Probab. Math. Statist. - 1991. - 44. - P. 53 - 59.

50. Dorogovtsev A. A. Stochastic integration and a class of Gaussian random processes // Ukr. Math. J. 1998ю - 50, № 4. - P. 550 - 561 .

51. Gelfand I. M., Vilenkin N. Ya. Generalized functions. - New York; London: Acad. Press, 1964. - 472 p.

52. Grigelionis B. Processes of Meixner type // Lith. Math. J. - 1999. - 39, № 1. - P. 33 - 41.

53. Grigelionis B. Generalized $z$-distributions and related stochastic processes // Ibid. - 2001. - 41, № 3. - P. 239-251.

54. Hida T. Analysis of Brownian functionals // Math. Lect. Notes. - 1975. - 13. - IV+61 p.

55. Hida T. Brownian motion. - New York etc.: Springer, 1980. $-\mathrm{XVI}+325 \mathrm{p}$.

56. Hida T., Kuo H. H., Potthoff J., Streit L. White Noise. An infinite dimensional calculus. - Dordrecht: Kluwer, 1993. - XIV+516 p.

57. Hitsuda M. Formula for Brownian partial derivatives // Proc. Second Japan-USSR Symp. Probab. Theory. - 1972. - P. $111-114$.

58. Huang Z. Y., Wu Y. Interacting Fock expansion of Lévy white noise functionals // Acta Appl. Math. 2004. - 82. - P. $333-352$.

59. Ito $Y$. Generalized Poisson functionals // Probab. Theory and Related Fields. - 1988. - 77. - P. $1-28$.

60. Ito Y., Kubo I. Calculus on Gaussian and Poisson white noises // Nagoya Math. J. - 1988. - 111. P. $41-84$.

61. Ji U. C., Obata N. A role of Bargmann-Segal spaces in characterization and expansion of operators on Fock space // J. Math. Soc. Jap. - 2004. - 56, № 2. - P. 311-338.

62. Ji U. C., Obata N., Ouerdiane H. Quantum Levy Laplacian and associated heat equation // J. Funct. Anal. - 2007. - 249, № 1. - P. 31 - 54 .

63. Kabanov Yu. M. Extended stochastic integrals // Teorija Verojatnostei i ee Pril. - 1975. - 20, № 4. P. $725-737$ (in Russian).

64. Kabanov Yu. M., Skorohod A. V. Extended stochastic integrals// Proc. School-Semin. Theory Stochast. Process. - Vilnus: Inst. Phys. Math., 1975. - P. 123-167 (in Russian).

65. Kachanovsky N. A. A generalized stochastic derivative connected with coloured noise measures // Meth Funct. Anal. and Top. - 2004. - 10, № 4. - P. 11-29.

66. Kachanovsky N. A. An extended stochastic integral and a Wick calculus on parametrized Kondratievtype spaces of Meixner white noise // Infinite Dimens. Anal. Quantum Probab. Relat. Top. - 2008. 11, № 4. - P. $541-564$.

67. Kachanovsky N. A. Biorthogonal Appell-like systems in a Hilbert space// Meth. Funct. Anal. and Top. - 1996. - № 3-4. - P. 36-52.

68. Kachanovsky N. A. Dual Appell-like systems and finite order spaces in non-Gaussian infinite dimensional analysis // Ibid. - 1998. - 4, № 2. - P. 41-52.

69. Kachanovsky N. A. Dual Appell system and Kondratiev spaces in analysis on Schwartz spaces // Ukr. Math. J. - 1997. - 49, № 4. - P. 581 - 589.

70. Kachanovsky N. A. Generalized stochastic derivatives on a space of regular generalized functions of Meixner white noise // Meth. Funct. Anal. and Top. - 2008. - 14, № 1. - P. 32 - 53.

71. Kachanovsky N.A. Generalized stochastic derivatives on parametrized spaces of regular generalized functions of Meixner white noise // Meth. Funct. Anal. and Top. - 2008. - 14, № 4. - P. 334 - 350. 
72. Kachanovsky N. A. Generalized stochastic derivatives on spaces of nonregular generalized functions of Meixner white noise // Ukr. Math. J. - 2008. - 60, № 6. - P. 848-875.

73. Kachanovsky N. A. On an extended stochastic integral and the Wick calculus on the connected with the generalized Meixner measure Kondratiev-type spaces // Meth. Funct. Anal. and Top. - 2007. - 13, № 4. - P. $338-379$.

74. Kachanovsky N.A. On analog of stochastic integral and Wick calculus in non-Gaussian infinite dimensional analysis // Ibid. - 1997. - 3, № 3. - P. 1 - 12 .

75. Kachanovsky N. A. On biorthogonal approach to construction of non-Gaussian analysis // Can. Math. Soc. Conf. Proc. - 2000. - 29. - P. 367-376.

76. Kachanovsky N.A. On the extended stochastic integral connected with the Gamma-measure on an infinite-dimensional space // Meth. Funct. Anal. and Top. - 2002. - 8, № 2. - P. 10-32.

77. Kachanovsky N. A. Pseudodifferential equations and a generalized translation operator in non-gaussian infinite-dimensional analysis // Ukr. Math. J. - 1999. - 51, № 10. - P. 1503 - 1511.

78. Kachanovsky N.A. Wick algebras on the spaces of coloured noise analysis and its application to quantum stochastic calculus // Spectral and Evolut. Problems. - 1999. - 9. - P. 138 - 145.

79. Kachanovsky N. A., Koshkin S. V. Minimality of Appell-like systems and embeddings of test function spaces in a generalization of white noise analysis // Meth. Funct. Anal. and Top. - 1999. - 5, № 3. P. $13-25$.

80. Kachanovsky N. A., Tesko V. A. Stochastic integral of Hitsuda-Skorohod type on the extended Fock space // Ukr. Math. J. - 2009. - 61, № 6. - P. 873-907.

81. Kondratiev $Y u$. G. A space of entire functions of infinite many variables that is connected with a rigging of a Fock space // Spectral Anal. Different. Operators. - Kiev: Inst. Math. Acad. Nauk Ukr. SSR, 1980 - P. 18-37 (in Russian)

82. Kondratiev $Y$. G. Nuclear spaces of entire functions in problems of infinite dimensional analysis // Dokl. AN SSSR. - 1980. - 254, № 6. - P. 1325 - 1329 (in Russian).

83. Kondratiev Yu. G. Wick powers of Gaussian random processes // Meth. Funct. Anal. Problems Math. Phys. - Kiev: Inst. Math. Acad. Nauk Ukr. SSR, 1978. - P. 129-158 (in Russian).

84. Kondratiev Yu. G., Lytvynov E. W. Operators of gamma white noise calculus // Infinite Dimens. Anal. Quantum Probab. Relat. Top. - 2000. - 3, № 3. - P. 303 - 335.

85. Kondratiev Yu. G., Samoilenko Yu. S. Generalized derivatives of probability measures on $\mathbb{R}^{\infty} / /$ Meth. Funct. Anal. Problems Math. Phys. - Kiev: Inst. Math. Acad. Nauk Ukr. SSR, 1978. - P. 159-176 (in Russian).

86. Kondratiev Yu. G., Samoilenko Yu. S. Integral representation of generalized positive-defined kernels of infinitely many variables // Dokl. AN SSSR. - 1976. - 227, № 4. - P. 800-803 (in Russian).

87. Kondratiev Yu. G., Samoilenko Yu. S. The spaces of trial and generalized functions of infinitely many variables // Rep. Math. Phys. - 1978. - 14, № 3. - P. $323-348$.

88. Kondratiev Yu. G., Silva L., Streit L. Generalized Appell systems // Meth. Funct. Anal. and Top. - 1997. - 3, № 3. - P. $28-61$.

89. Kondratiev Yu. G., Silva L., Streit L., Us G. F. Analysis on Poisson and Gamma spaces // Infinite Dimens. Anal. Quantum Probab. Relat. Top. - 1998. - 1, № 1. - P. 91 - 117.

90. Kondratiev Yu. G., Streit L. Spaces of white noise distributions: Constructions, descriptions, applications // Rep. Math. Phys. - 1993. - 33. - P. 341-366.

91. Kondratiev Yu. G., Streit L., Westercamp W., Yan J. Generalized functions in infnite-dimensional analysis // Hiroshima Math. J. - 1998. - 28. - P. 213-260.

92. V. D. Koshmanenko, Samoilenko Yu. S. Isomorphism of Fock space with a space of functions of infinitely many variables // Ukr. Math. J. - 1975. - 27, № 5. - P. 552 - 555.

93. Kubo I., Kuо H. H., Sengupta A. N. White noise analysis on a new space of Hida distributions // Infinite Dimens. Anal. Quantum Probab. Relat. Top. - 1999. - 2, № 3. - P. 315-335.

94. T. Lindstrom, Oksendal B., J. Uboe Wick multiplication and Itô-Skorohod stochastic differential equations // Ideas and Meth. Math. Anal., Stochastics and Appl. - Cambridge Univ. Press, 1992. P. $183-206$.

95. Lytvynov E. W. Multiple Wiener integrals and non-Gaussian white noise analysis: a Jacobi field approach // Meth. Funct. Anal. and Top. - 1995. - 1, № 1. - P. $61-85$.

96. Lytvynov E. W. Orthogonal decompositions for Lévy processes with an application to the Gamma, Pascal, and Meixner processes // Infinite Dimens. Anal. Quantum Probab. Relat. Top. - 2003. - 6, № 1. - P. $73-102$. 
97. Lytvynov E. W. Polynomials of Meixner's type in infinite dimensions - Jacobi fields and orthogonality measures // J. Funct. Anal. - 2003. - 200, № 1. - P. $118-149$.

98. J. Meixner Orthogonale Polynomsysteme mit einer besonderen Gestalt der erzeugen-den Funktion // J. London Math. Soc. - 1934. - 9, № 1. - S. 6-13.

99. Norin N. V. An extended stochastic integral for non-Gaussian measures in local-convex spaces // Uspehi Math. Nauk. - 1986. - 41, № 3. - S. 199-200 (in Russian).

100. Norin N. V. Stochastic integrals and differentiable measures // Teorija Verojatnostei i ee Pril. - 1987. 32, № 1. - S. 114-124 (in Russian).

101. Nualart D., Schoutens W. Chaotic and predictable representations for Lévy processes // Stochast Process. and Appl. - 2000. - 90. - P. 109-122.

102. Obata $N$. Inverse $S$-transform, Wick product and overcompleteness of exponential vectors. Quantum information. - River Edge, NJ: World Sci. Publ., 2002. - Vol. 4. - P. 147-176.

103. Obata N. Unitarity criterion in white noise calculus and nonexistence of unitary evolutions driven by higher powers of quantum white noises // Stochastic models, II (Spanish), Aportaciones Mat. Investig., Soc. Mat. Mexicana. - Mexico, 2001. - Vol. 16. - P. 251-269.

104. Obata N. Wick product of white noise operators and quantum stochastic differential equations // J Math. Soc. Jap. - 1999. - 51, № 3. - P. 613-641.

105. Ouerdiane $H$. Algebres nucleaires de fonctions entieres et equations aux derivees partielles stochastiques // Nagoya Math. J. - 1998. - 151. - P. 107-127.

106. Ouerdiane H. Distributions gaussiennes et applications aux equations aux derivees partielles stochastiques // Math. Phys. and Stochast. Anal. - River Edge, NJ: World Sci. Publ., 2000. - P. 318-331.

107. Pylypenko A. Yu. On the properties of an operator of stochastic differentiation constructed on a group // Ukr. Math. J. - 1996. - 48, № 4. - P. 623-630.

108. Rodionova $I$. $V$. Analysis connected with generating functions of exponential type in one and infinite dimensions // Meth. Funct. Anal. and Top. - 2005. - 11, № 3. - P. 275-297.

109. Skorohod A. V. Integration in Hilbert Space. - Berlin: Springer, 1974. - $230 \mathrm{p}$.

110. Skorohod A. V. On a generalization of a stochastic integral // Teorija Verojatnostei i ee Pril. - 1975. 20, № 2. - S. 223-238 (in Russian).

111. Smolianov $O . G$. Differentiable measures on the group of functions taking values in a compact Ide group // Abstr. 6-th Int. Vilnius Conf. Probab. Theory and Math. Stat. - Vilnius, 1993. - P. 139-140.

112. Tesko V. A. Spaces appearing in the construction of infinite-dimensional analysis according to the biorthogonal scheme // Ukr. Math. J. - 2004. - 56, № 7. - P. 1166-1181.

113. Tsilevich N. V., Vershik A. M., Yor M. An infinite-dimensional analogue of the Lebesgue measure and distinguished properties of the gamma process // J. Funct. Anal. - 2001. - 185, № 1. - P. $274-296$.

114. Tsilevich N. V., Vershik A. M., Yor M. The Markov-Krein identity and quasiinvariance of the gammaprocess // Notes Sankt-Peterburg office V. A. Steklov Math. Inst. - 2001. - 283. - P. 21 - 36 (in Russian)

115. Tsilevich N. V., Vershik A. M. Quasi-invariance of the gamma process and multiplicative properties of the Poisson-Dirichlet measures // C. r. Acad. sci. Ser. I Math. - 1999. - 2. - P. 163-168.

116. Us G. F. Dual Appell systems in Poissonian analysis // Meth. Funct. Anal. and Top. - 1995. - 1, № 1 . - P. $93-108$.

117. Us G. F. Towards a coloured noise analysis // Ibid. - 1997. - 3, № 2. - P. 83-99.

118. Yablonsky E. Characterization of operators on a Kondratiev space in the non-Gaussian setting: biorthogonal approach // Infinite Dimens. Anal. Quantum Probab. Relat. Top. - 2005. - 8, № 3. P. $439-452$.

Одержано 30.03.10 